MATHEMATICS OF COMPUTATION

Volume 71, Number 240, Pages 1609-1640

S $0025-5718(02) 01420-5$

Article electronically published on March 20, 2002

\title{
ON THE STEP-BY-STEP CONSTRUCTION OF QUASI-MONTE CARLO INTEGRATION RULES THAT ACHIEVE STRONG TRACTABILITY ERROR BOUNDS IN WEIGHTED SOBOLEV SPACES
}

\author{
I. H. SLOAN, F. Y. KUO, AND S. JOE
}

\begin{abstract}
We develop and justify an algorithm for the construction of quasiMonte Carlo (QMC) rules for integration in weighted Sobolev spaces; the rules so constructed are shifted rank-1 lattice rules. The parameters characterising the shifted lattice rule are found "component-by-component": the $(d+1)$-th component of the generator vector and the shift are obtained by successive 1-dimensional searches, with the previous $d$ components kept unchanged. The rules constructed in this way are shown to achieve a strong tractability error bound in weighted Sobolev spaces. A search for $n$-point rules with $n$ prime and all dimensions 1 to $d$ requires a total cost of $O\left(n^{3} d^{2}\right)$ operations. This may be reduced to $O\left(n^{3} d\right)$ operations at the expense of $O\left(n^{2}\right)$ storage. Numerical values of parameters and worst-case errors are given for dimensions up to 40 and $n$ up to a few thousand. The worst-case errors for these rules are found to be much smaller than the theoretical bounds.
\end{abstract}

\section{INTRODUCTION}

In this paper we develop a constructive approach to the design of quasi-Monte Carlo (or QMC) integration rules for approximating integrals over the $d$-dimensional unit cube,

$$
I_{d}(f)=\int_{[0,1]^{d}} f(\boldsymbol{x}) \mathrm{d} \boldsymbol{x} .
$$

We recall that QMC rules are algorithms of the form

$$
Q_{n, d}(f)=\frac{1}{n} \sum_{i=0}^{n-1} f\left(\boldsymbol{x}_{i}\right),
$$

with the points $\boldsymbol{x}_{i}=\boldsymbol{x}_{n, d, i} \in[0,1]^{d}$ for $i=0, \ldots, n-1$ chosen in some deterministic way.

Recently the tractability of this problem in the worst-case setting has been studied by Sloan and Woźniakowski [8] in weighted Sobolev spaces of functions with square-integrable mixed first derivatives. Following [8], the problem is said to be QMC tractable if the minimal number $n_{0}(\varepsilon, d)$ of quadrature points $\boldsymbol{x}_{i}$ needed in (11.1) to reduce the worst-case error from its initial value by a factor $\varepsilon>0$ is bounded by a polynomial in $\varepsilon^{-1}$ and $d$. The problem is said to be strongly QMC tractable

Received by the editor October 30, 2000.

2000 Mathematics Subject Classification. Primary 65D30, 65D32; Secondary 68Q25.

(C)2002 American Mathematical Society 
if that bound is independent of $d$. In this situation, the least possible value (or the infimum) of the power of $\varepsilon^{-1}$ is called the $\varepsilon$-exponent.

In 8 ] it is shown that the necessary and sufficient condition for strong QMC tractability is

$$
\sum_{j=1}^{\infty} \gamma_{j}<\infty
$$

where the nonincreasing sequence $\left\{\gamma_{j}\right\}$ of positive numbers $\gamma_{j}$ (the "weights") characterizes the decreasing importance of the successive co-ordinate directions in the weighted Sobolev space. (Precise details of the space are given in Section 2) If this condition holds, then $n_{0}(\varepsilon, d)$ is bounded by $C \varepsilon^{-2}$, with $C$ independent of $d$; thus the $\varepsilon$-exponent is at most 2 .

More general quadrature formulas are considered in [10] and [4]: the former allows any positive weights, the latter allows even negative weights. The conclusion in both cases is that the greater freedom does not make the problem any easier, in the sense that (1.2) is still the necessary and sufficient condition for strong tractability.

The proof used in $[8$ is nonconstructive (the argument involves averaging over all choices of the quadrature points $\boldsymbol{x}_{i}$ in (1.1)), and therefore gives no clue as to how to construct QMC rules that achieve an error bound appropriate to strong QMC tractability.

The spur to the present construction comes from a later paper [9], where it is proved that if (1.2) holds then the strong tractability bound can be achieved, if $n$ is prime, by QMC rules from the relatively small class of "shifted lattice rules". These are rules of the form (given that $n$ is prime)

$$
Q_{n, d}(f)=\frac{1}{n} \sum_{i=0}^{n-1} f\left(\left\{\frac{i \boldsymbol{z}}{n}+\boldsymbol{\Delta}\right\}\right)
$$

where $\boldsymbol{z} \in \mathbb{Z}^{d}$ has no component divisible by $n$, and $\boldsymbol{\Delta} \in[0,1)^{d}$ is the "shift". Here the braces around a vector indicate that we take the fractional part of each component of the vector. (The paper [9] also gives the improved value of 1 for the $\varepsilon$-exponent if the weights satisfy $\sum_{j=1}^{\infty} \gamma_{j}^{1 / 2}<\infty$, thereby confirming results previously obtained nonconstructively by [3]; we do not obtain results of this kind here.)

Lattice rules, discussed in [5] and [6], are usually considered only in the context of periodic functions, but in Section 6 of [9] and here they are considered for the integration of general (nonperiodic) functions from the weighted Sobolev space.

The total number of distinct lattice rules with $n$ quadrature points is finite, since it is clearly sufficient in (1.3) to restrict $\boldsymbol{z}$ to the set $\mathbb{Z}_{n}^{d}$, where

$$
\mathbb{Z}_{n}:=\{1,2, \ldots, n-1\} .
$$

However, a direct search for good QMC rules of the form (1.3) is infeasible when $d$ is large, because the number of choices of the integer vector $\boldsymbol{z}$ is $(n-1)^{d}$, which rises exponentially with $d$. In addition, there is the problem of choosing the $d$ dimensional shift $\boldsymbol{\Delta}$.

In the present paper we develop a theoretical framework for the practical construction of shifted lattice rules that achieve the predicted strong tractability error bound. 
Our construction is inspired by a component-by-component construction of "good" lattice rules introduced, for the classical context of periodic functions, in the recent paper 7]. At each stage of that construction a one-dimensional search is carried out for the last component of $\boldsymbol{z}$, with all previously computed components held fixed, in the knowledge that there exists at least one choice for the added component of $\boldsymbol{z}$ for which the desired error bound is achieved. From this it follows that the best possible choice must certainly achieve the bound.

The present setting is different from that in [7, because the spaces are now Sobolev spaces instead of Korobov spaces, and there is the extra difficulty of having to find a suitable shift $\boldsymbol{\Delta}$ in (1.3). Nevertheless, the final result (stated in Theorem 4.4) has a similar flavor: in essence if $\boldsymbol{z} \in \mathbb{Z}_{n}^{d}$ and $\boldsymbol{\Delta} \in[0,1)^{d}$ are such as to achieve an appropriate bound for the worst-case error in the $d$-dimensional case, then there exists a pair $\left(z_{d+1}, \Delta_{d+1}\right) \in \mathbb{Z}_{n} \times[0,1)$ for which the $(d+1)$-dimensional case with these components adjoined (and all other components left unchanged) satisfies an analogous bound. It follows that a suitable pair $\left(z_{d+1}, \Delta_{d+1}\right)$ could in principle be obtained by minimizing the worst-case error in the $(d+1)$-dimensional case over all possible choices of the pair. Even that task would be difficult. Fortunately, the theorem also establishes that a satisfactory pair $\left(z_{d+1}, \Delta_{d+1}\right)$ can be obtained by finding first $z_{d+1}$ (by minimizing the explicitly known mean square error over all 1-dimensional shifts $\Delta_{d+1}$ ) and then, with $z_{d+1}$ fixed, determining the shift by minimizing the worst-case error over $\Delta_{d+1}$. The theorem even states that the search over the shifts $\Delta_{d+1}$ can be restricted to the discrete set $\{1 /(2 n), 3 /(2 n), \ldots$, $(2 n-1) /(2 n)\}$.

The final fruit of the theoretical considerations is the algorithm in Section 5 This gives a foolproof way of constructing, for each prime value of $n$, an unending sequence of shifted lattice rules $Q_{n, d}$, with $d$ increasing, at the cost of carrying out two 1-dimensional searches of length $n$ at each stage of the construction. The total cost for constructing all rules up to dimension $d$ is at most $O\left(n^{3} d^{2}\right)$ operations. This may be reduced to $O\left(n^{3} d\right)$ operations at the expense of $O\left(n^{2}\right)$ storage. The quadrature error is bounded by $\left|I_{d}(f)-Q_{n, d}(f)\right| \leq C / n^{1 / 2}$, with a known value of $C$, independent of $d$.

Explicit examples of such sequences are shown in Section 6 for $n=1009,2003$ and 4001 (all of which are prime). The numerical results reveal that the resulting worst-case errors are always much smaller than the theoretical bounds. Moreover, the apparent dependence of the worst-case errors on $n$ is always better than the predicted $O\left(n^{-1 / 2}\right)$.

\section{Weighted Sobolev SPACes}

We start by defining a 1-dimensional Sobolev space which is parametrized by positive numbers $\beta$ and $\gamma$ and a number $a \in[0,1]$. The Hilbert space $H_{1}=H_{1, \beta, \gamma, a}$ is the space of absolutely continuous and once-differentiable real functions defined on $[0,1]$ with square-integrable first derivatives. The inner product in $H_{1}$ has the form

$$
\langle f, g\rangle_{1}=\langle f, g\rangle_{1, \beta, \gamma, a}:=\beta^{-1} f(a) g(a)+\gamma^{-1} \int_{0}^{1} f^{\prime}(x) g^{\prime}(x) \mathrm{d} x
$$

with corresponding norm $\|f\|_{1}=\sqrt{\langle f, f\rangle_{1}}$. Though we may assume that $\beta=1$ as in [9] (there it is also assumed that $a=1$ ), we shall not make this assumption since there is no disadvantage in allowing $\beta$ to take on any positive value. A general choice 
of the parameter $a$ is also considered in 4 . The space $H_{1}$ is a reproducing kernel Hilbert space, since there exists a (unique) $K_{1}(x, y)$ such that $f(y)=\left\langle f, K_{1}(\cdot, y)\right\rangle_{1}$ for all $f \in H_{1}$. An explicit expression for this reproducing kernel is given in the next lemma.

Lemma 2.1. The reproducing kernel for the space $H_{1}$ is

$$
K_{1}(x, y)=K_{1, \beta, \gamma, a}(x, y)=\beta+\gamma \mu_{a}(x, y)
$$

where

$$
\mu_{a}(x, y)= \begin{cases}\min (|x-a|,|y-a|), & \text { if }(x-a)(y-a)>0, \\ 0, & \text { if }(x-a)(y-a) \leq 0 .\end{cases}
$$

The proof of this lemma is similar to the proof in [4] for the case when $\beta=1$, and hence is omitted.

For the functional of one-dimensional integration,

$$
I_{1}(f)=\int_{0}^{1} f(x) \mathrm{d} x
$$

we can easily find its representer, that is, the function $h_{1} \in H_{1}$ such that $\left\langle f, h_{1}\right\rangle_{1}=$ $I_{1}(f)$.

Lemma 2.2. The representer of integration in the space $H_{1}$ is

$$
h_{1}(y)=h_{1, \beta, \gamma, a}(y)=\beta+\gamma \omega_{a}(y),
$$

where

$$
\omega_{a}(y)= \begin{cases}(y-a)\left(1-\frac{a}{2}-\frac{y}{2}\right), & \text { if } y>a \\ (a-y)\left(\frac{a}{2}+\frac{y}{2}\right), & \text { if } y \leq a\end{cases}
$$

The norm of this representer is given by

$$
\left\|h_{1, \beta, \gamma, a}\right\|_{1}=\left[\beta+\gamma\left(a^{2}-a+\frac{1}{3}\right)\right]^{\frac{1}{2}} .
$$

Again, the proof of this lemma is similar to the proof in [4] for the case when $\beta=1$.

Now we define our $d$-dimensional weighted Sobolev space parametrized by a vector $\boldsymbol{a} \in[0,1]^{d}$. Suppose we have two positive sequences $\boldsymbol{\beta}=\left\{\beta_{j}\right\}$ and $\boldsymbol{\gamma}=\left\{\gamma_{j}\right\}$. We define the $d$-dimensional reproducing kernel Hilbert space $H_{d}$ as the tensor product

$$
H_{d}=H_{d, \boldsymbol{\beta}, \boldsymbol{\gamma}, \boldsymbol{a}}:=H_{1, \beta_{1}, \gamma_{1}, a_{1}} \otimes H_{1, \beta_{2}, \gamma_{2}, a_{2}} \otimes \cdots \otimes H_{1, \beta_{d}, \gamma_{d}, a_{d}}
$$

of $d$ different 1-dimensional Hilbert spaces with different weights. The inner product in the space $H_{d}$ is given by

$$
\langle f, g\rangle_{d}=\sum_{\mathfrak{u} \subset \mathcal{D}}\left(\prod_{j \notin \mathfrak{u}} \beta_{j}^{-1}\right)\left(\prod_{j \in \mathfrak{u}} \gamma_{j}^{-1}\right) \int_{[0,1]^{|\mathfrak{u}|}} \frac{\partial^{|\mathfrak{u}|}}{\partial \boldsymbol{x}_{\mathfrak{u}}} f\left(\boldsymbol{x}_{\mathfrak{u}}, \boldsymbol{a}\right) \frac{\partial^{|\mathfrak{u}|}}{\partial \boldsymbol{x}_{\mathfrak{u}}} g\left(\boldsymbol{x}_{\mathfrak{u}}, \boldsymbol{a}\right) \mathrm{d} \boldsymbol{x}_{\mathfrak{u}}
$$

where $\mathcal{D}=\{1,2, \ldots, d\},\left(\boldsymbol{x}_{\mathfrak{u}}, \boldsymbol{a}\right)$ is a $d$-dimensional vector whose $j$-th component is $x_{j}$ if $j \in \mathfrak{u}$ and $a_{j}$ if $j \notin \mathfrak{u}$, and $\mathrm{d} \boldsymbol{x}_{\mathfrak{u}}=\prod_{j \in \mathfrak{u}} \mathrm{d} x_{j}$. The corresponding norm in $H_{d}$ 
is given by $\|f\|_{d}=\sqrt{\langle f, f\rangle_{d}}$. The reproducing kernel in the space $H_{d}$ is given by

$$
K_{d}(\boldsymbol{x}, \boldsymbol{y})=K_{d, \boldsymbol{\beta}, \boldsymbol{\gamma}, \boldsymbol{a}}(\boldsymbol{x}, \boldsymbol{y})=\prod_{j=1}^{d}\left(\beta_{j}+\gamma_{j} \mu_{a_{j}}\left(x_{j}, y_{j}\right)\right) .
$$

For the $d$-dimensional integral functional

$$
I_{d}(f)=\int_{[0,1]^{d}} f(\boldsymbol{x}) \mathrm{d} \boldsymbol{x},
$$

its representer in $H_{d}$ is given by

$$
h_{d}(\boldsymbol{y})=h_{d, \boldsymbol{\beta}, \boldsymbol{\gamma}, \boldsymbol{a}}(\boldsymbol{y})=\prod_{j=1}^{d} h_{1, \beta_{j}, \gamma_{j}, a_{j}}\left(y_{j}\right)=\prod_{j=1}^{d}\left(\beta_{j}+\gamma_{j} \omega_{a_{j}}\left(y_{j}\right)\right),
$$

where $\omega_{a_{j}}\left(y_{j}\right)$ is given by (2.2) with $a=a_{j}$ and $y=y_{j}$. By the definition of the norm corresponding to the inner product in (2.3), we can show that the norm of the representer is

$$
\left\|h_{d}\right\|_{d}=\prod_{j=1}^{d}\left\|h_{1, \beta_{j}, \gamma_{j}, a_{j}}\right\|_{1}=\prod_{j=1}^{d}\left[\beta_{j}+\gamma_{j}\left(a_{j}^{2}-a_{j}+\frac{1}{3}\right)\right]^{\frac{1}{2}} .
$$

Let $P_{n, d}=\left\{\boldsymbol{x}_{0}, \ldots, \boldsymbol{x}_{n-1}\right\}$ be the set of points for the QMC rule (1.1). Then for the Hilbert space $H_{d}$ with reproducing kernel $K_{d}$, we define the worst-case error $e_{n, d}\left(P_{n, d}, K_{d}\right)$ by

$$
e_{n, d}\left(P_{n, d}, K_{d}\right):=\sup \left\{\left|I_{d}(f)-Q_{n, d}(f)\right|: f \in H_{d},\|f\|_{d} \leq 1\right\} .
$$

Since

$$
I_{d}(f)-Q_{n, d}(f)=\left\langle f, h_{d}-\frac{1}{n} \sum_{i=0}^{n-1} K_{d}\left(\cdot, \boldsymbol{x}_{i}\right)\right\rangle_{d},
$$

it follows that

$$
e_{n, d}\left(P_{n, d}, K_{d}\right)=\left\|h_{d}-\frac{1}{n} \sum_{i=0}^{n-1} K_{d}\left(\cdot, \boldsymbol{x}_{i}\right)\right\|_{d}
$$

We also define the initial approximation $Q_{0, d}(f)$ to be 0 , so that the initial worstcase error is simply $e_{0, d}\left(K_{d}\right)=\left\|h_{d}\right\|_{d}$.

Lemma 2.3. The square worst-case error $e_{n, d}^{2}\left(P_{n, d}, K_{d}\right)$ with $K_{d}=K_{d, \boldsymbol{\beta}, \boldsymbol{\gamma}, \boldsymbol{a}}$ may be written as

$$
\begin{aligned}
\prod_{j=1}^{d}[ & \left.\beta_{j}+\gamma_{j}\left(a_{j}^{2}-a_{j}+\frac{1}{3}\right)\right]-\frac{2}{n} \sum_{i=0}^{n-1} \prod_{j=1}^{d}\left[\beta_{j}+\gamma_{j} \omega_{a_{j}}\left(x_{i, j}\right)\right] \\
+ & \frac{1}{n^{2}} \sum_{i=0}^{n-1} \sum_{k=0}^{n-1} \prod_{j=1}^{d}\left[\beta_{j}+\gamma_{j} \mu_{a_{j}}\left(x_{i, j}, x_{k, j}\right)\right],
\end{aligned}
$$

where $\omega_{a}$ and $\mu_{a}$ are given by (2.2) and (2.1) respectively, and $x_{i, j}$ is the $j$-th component of $\boldsymbol{x}_{i}$. 
Proof. It follows from (2.7) that

$$
\begin{aligned}
e_{n, d}^{2}\left(P_{n, d}, K_{d}\right)= & \left\langle h_{d}, h_{d}\right\rangle_{d}-\frac{2}{n} \sum_{i=0}^{n-1}\left\langle h_{d}, K_{d, \boldsymbol{\beta}, \boldsymbol{\gamma}, \boldsymbol{a}}\left(\cdot, \boldsymbol{x}_{i}\right)\right\rangle_{d} \\
& +\frac{1}{n^{2}} \sum_{i=0}^{n-1} \sum_{k=0}^{n-1}\left\langle K_{d, \boldsymbol{\beta}, \boldsymbol{\gamma}, \boldsymbol{a}}\left(\boldsymbol{x}_{i}, \cdot\right), K_{d, \boldsymbol{\beta}, \boldsymbol{\gamma}, \boldsymbol{a}}\left(\cdot, \boldsymbol{x}_{k}\right)\right\rangle_{d} \\
= & \left\|h_{d}\right\|_{d}^{2}-\frac{2}{n} \sum_{i=0}^{n-1} h_{d}\left(\boldsymbol{x}_{i}\right)+\frac{1}{n^{2}} \sum_{i=0}^{n-1} \sum_{k=0}^{n-1} K_{d, \boldsymbol{\beta}, \boldsymbol{\gamma}, \boldsymbol{a}}\left(\boldsymbol{x}_{i}, \boldsymbol{x}_{k}\right) .
\end{aligned}
$$

The result now follows from (2.4), (2.5), and (2.6).

When all the $\beta_{j}$ are 1 and all of the components of $\boldsymbol{a}$ are also 1 , then the corresponding worst-case error $e_{n, d}\left(P_{n, d}, K_{d}\right)$ is simply the weighted version of the $L_{2}$ star discrepancy considered in earlier work (for example, see [2] and [8]). When all the $\beta_{j}$ are 1 and all of the components of $\boldsymbol{a}$ are $1 / 2$, then the corresponding worst-case error $e_{n, d}\left(P_{n, d}, K_{d}\right)$ is the weighted version of the $L_{2}$ centred discrepancy discussed in [1].

\section{THE SHIFT-INVARIANT KERNEL}

In the subsequent work we shall need the "shift-invariant" kernel corresponding to (2.4), as defined in Hickernell and Woźniakowski [3]. A shift-invariant kernel associated with any reproducing kernel $K_{d}(\boldsymbol{x}, \boldsymbol{y})$ is defined by

$$
K_{d}^{*}(\boldsymbol{x}, \boldsymbol{y}):=\int_{[0,1]^{d}} K_{d}(\{\boldsymbol{x}+\boldsymbol{\Delta}\},\{\boldsymbol{y}+\boldsymbol{\Delta}\}) \mathrm{d} \boldsymbol{\Delta} .
$$

It is said to be shift-invariant because

$$
K_{d}^{*}(\boldsymbol{x}, \boldsymbol{y})=K_{d}^{*}(\{\boldsymbol{x}+\boldsymbol{\Delta}\},\{\boldsymbol{y}+\boldsymbol{\Delta}\}), \quad \boldsymbol{x}, \boldsymbol{y}, \boldsymbol{\Delta} \in[0,1]^{d} .
$$

Lemma 3.1. The shift-invariant kernel associated with the reproducing kernel $K_{1, \beta, \gamma, a}$ of Lemma 2.1] is given by

$$
K_{1, \beta, \gamma, a}^{*}(x, y)=\beta+\gamma\left(|x-y|^{2}-|x-y|+a^{2}-a+\frac{1}{2}\right), x, y \in[0,1) .
$$

The proof of this lemma in the case when $a=1$ can be found in [3]. The proof when $a \in[0,1)$ is not difficult but is tedious, and hence is omitted.

The shift-invariant kernel $K_{d, \boldsymbol{\beta}, \boldsymbol{\gamma}, \boldsymbol{a}}^{*}$ associated with the reproducing kernel $K_{d, \boldsymbol{\beta}, \boldsymbol{\gamma}, \boldsymbol{a}}$ in (2.4) is then given by

$$
\begin{aligned}
K_{d, \boldsymbol{\beta}, \boldsymbol{\gamma}, \boldsymbol{a}}^{*}(\boldsymbol{x}, \boldsymbol{y}) & =\int_{[0,1]^{d}} K_{d, \boldsymbol{\beta}, \boldsymbol{\gamma}, \boldsymbol{a}}(\{\boldsymbol{x}+\boldsymbol{\Delta}\},\{\boldsymbol{y}+\boldsymbol{\Delta}\}) \mathrm{d} \boldsymbol{\Delta} \\
& =\prod_{j=1}^{d} K_{1, \beta_{j}, \gamma_{j}, a_{j}}^{*}\left(x_{j}, y_{j}\right) \\
& =\prod_{j=1}^{d}\left[\beta_{j}+\gamma_{j}\left(\left|x_{j}-y_{j}\right|^{2}-\left|x_{j}-y_{j}\right|+a_{j}^{2}-a_{j}+\frac{1}{2}\right)\right] .
\end{aligned}
$$


Generalized weighted Korobov spaces are spaces in which the reproducing kernels are of the form

$$
\prod_{j=1}^{d}\left[\hat{\beta}_{j}+\hat{\gamma}_{j} \sum_{h=-\infty}^{\infty} \frac{e^{2 \pi \mathrm{i} h\left(x_{j}-y_{j}\right)}}{|h|^{\alpha}}\right], \quad \alpha>1,
$$

where the prime in the sum indicates that we exclude the $h=0$ term. Such spaces have been considered previously in [3] and [9]. In the following lemma, we show that $K_{d, \boldsymbol{\beta}, \boldsymbol{\gamma}, \boldsymbol{a}}^{*}$ is a kernel for such a weighted Korobov space with $\alpha=2$.

Lemma 3.2. The kernel $K_{d, \boldsymbol{\beta}, \boldsymbol{\gamma}, \boldsymbol{a}}^{*}$ of (3.1) may be written as

$$
K_{d, \boldsymbol{\beta}, \boldsymbol{\gamma}, \boldsymbol{a}}^{*}(\boldsymbol{x}, \boldsymbol{y})=\prod_{j=1}^{d}\left[\hat{\beta}_{j}+\hat{\gamma}_{j} \sum_{h=-\infty}^{\infty} \frac{e^{2 \pi \mathrm{i} h\left(x_{j}-y_{j}\right)}}{h^{2}}\right],
$$

where

$$
\hat{\beta}_{j}=\beta_{j}+\gamma_{j}\left(a_{j}^{2}-a_{j}+\frac{1}{3}\right) \quad \text { and } \quad \hat{\gamma}_{j}=\frac{\gamma_{j}}{2 \pi^{2}} .
$$

Proof. We have from Lemma 3.1

$$
\begin{aligned}
K_{1, \beta, \gamma, a}^{*}(x, y) & =\beta+\gamma\left(|x-y|^{2}-|x-y|+a^{2}-a+\frac{1}{2}\right) \\
& =\beta+\gamma\left(B_{2}(|x-y|)+a^{2}-a+\frac{1}{3}\right),
\end{aligned}
$$

where $B_{2}$ is the Bernoulli polynomial given by $B_{2}(x)=x^{2}-x+\frac{1}{6}$. We can write $B_{2}$ as the Fourier expansion (for example, see [6])

$$
B_{2}(x)=\frac{1}{2 \pi^{2}} \sum_{h=-\infty}^{\infty} \frac{e^{2 \pi \mathrm{i} h x}}{h^{2}}, \quad x \in[0,1] .
$$

It then follows that we can write $K_{1, \beta, \gamma, a}^{*}$ as

$$
\begin{aligned}
K_{1, \beta, \gamma, a}^{*}(x, y) & =\beta+\gamma\left(\frac{1}{2 \pi^{2}} \sum_{h=-\infty}^{\infty} \frac{e^{2 \pi \mathrm{i} h|x-y|}}{h^{2}}+a^{2}-a+\frac{1}{3}\right) \\
& =\beta+\gamma\left(a^{2}-a+\frac{1}{3}\right)+\frac{\gamma}{2 \pi^{2}} \sum_{h=-\infty}^{\infty} \frac{e^{2 \pi \mathrm{i} h|x-y|}}{h^{2}} .
\end{aligned}
$$

Hence

$$
\begin{aligned}
K_{d, \boldsymbol{\beta}, \boldsymbol{\gamma}, \boldsymbol{a}}^{*}(\boldsymbol{x}, \boldsymbol{y}) & =\prod_{j=1}^{d} K_{1, \beta_{j}, \gamma_{j}, a_{j}}^{*}\left(x_{j}, y_{j}\right) \\
& =\prod_{j=1}^{d}\left[\beta_{j}+\gamma_{j}\left(a_{j}^{2}-a_{j}+\frac{1}{3}\right)+\frac{\gamma_{j}}{2 \pi^{2}} \sum_{h=-\infty}^{\infty} \frac{e^{2 \pi \mathrm{i} h\left|x_{j}-y_{j}\right|}}{h^{2}}\right] .
\end{aligned}
$$

Since

$$
\sum_{h=-\infty}^{\infty} \frac{e^{2 \pi \mathrm{i} h\left(x_{j}-y_{j}\right)}}{h^{2}}=\sum_{h=-\infty}^{\infty} \frac{e^{2 \pi \mathrm{i} h\left(y_{j}-x_{j}\right)}}{h^{2}},
$$

this completes the proof. 


\section{A theORETiCAL FOUNDATION FOR COMPONENT-BY-COMPONENT SEARCHES}

We consider "shifted" rank-1 lattice rules, that is, quasi-Monte Carlo rules with quadrature points given by the set

$$
P_{n, d}(\boldsymbol{z}, \boldsymbol{\Delta}):=\left\{\left\{\frac{i \boldsymbol{z}}{n}+\boldsymbol{\Delta}\right\}: i=0,1, \ldots, n-1\right\},
$$

where $\boldsymbol{\Delta} \in[0,1)^{d}$ is the "shift" of the lattice $\{i \boldsymbol{z} / n\}, i=0,1, \ldots, n-1$, and $\boldsymbol{z} \in \mathbb{Z}_{n}^{d}$ is the generator vector with $\mathbb{Z}_{n}$ given by (1.4). For simplicity, $n$ is chosen to be a prime number and hence $n>1$.

The following theorem asserts the existence of a shifted lattice rule with relatively small worst-case error.

Theorem 4.1. Let $n$ be a prime number and $K_{d}=K_{d, \boldsymbol{\beta}, \boldsymbol{\gamma}, \boldsymbol{a}}$. Then there exist a choice of $\boldsymbol{z} \in \mathbb{Z}_{n}^{d}$ and a choice of $\boldsymbol{\Delta} \in[0,1)^{d}$ such that

$$
\begin{aligned}
e_{n, d}^{2}\left(P_{n, d}(\boldsymbol{z}, \boldsymbol{\Delta}), K_{d}\right) & \leq \frac{1}{n} \prod_{j=1}^{d}\left(\beta_{j}+\gamma_{j}\left(a_{j}^{2}-a_{j}+\frac{1}{2}\right)\right) \\
& \leq \frac{1}{n} \prod_{j=1}^{d}\left(\beta_{j}+\frac{\gamma_{j}}{2}\right) .
\end{aligned}
$$

Proof. Let $K_{d}^{*}=K_{d, \boldsymbol{\beta}, \boldsymbol{\gamma}, \boldsymbol{a}}^{*}$ denote the shift-invariant kernel associated with $K_{d}=$ $K_{d, \boldsymbol{\beta}, \boldsymbol{\gamma}, \boldsymbol{a}}$, given explicitly by Lemma 3.2. Then it is known from [3, formula (37)] that

$$
\int_{[0,1]^{d}} e_{n, d}^{2}\left(P_{n, d}(\boldsymbol{z}, \boldsymbol{\Delta}), K_{d}\right) \mathrm{d} \boldsymbol{\Delta}=e_{n, d}^{2}\left(P_{n, d}(\boldsymbol{z}), K_{d}^{*}\right)
$$

where

$$
P_{n, d}(\boldsymbol{z}):=\left\{\left\{\frac{i \boldsymbol{z}}{n}\right\}: i=0,1, \ldots, n-1\right\}=P_{n, d}(\boldsymbol{z}, \mathbf{0}) .
$$

Thus for an arbitrary fixed vector $\boldsymbol{z} \in \mathbb{Z}_{n}^{d}$ there exists at least one shift $\boldsymbol{\Delta} \in[0,1)^{d}$ such that

$$
e_{n, d}^{2}\left(P_{n, d}(\boldsymbol{z}, \boldsymbol{\Delta}), K_{d}\right) \leq e_{n, d}^{2}\left(P_{n, d}(\boldsymbol{z}), K_{d}^{*}\right) .
$$

Now recall that $K_{d}^{*}$ is given by Lemma 3.2 Let

$$
\zeta(p)=\sum_{h=1}^{\infty} h^{-p}, \quad p>1,
$$

be the Riemann zeta function. A slight adaptation of Lemma 2(ii) in [9] shows that if $n$ satisfies, for all $j \geq 1$,

$$
\begin{aligned}
n & \geq 1+\frac{\hat{\gamma}_{j}}{\hat{\beta}_{j}} \zeta(2) \\
& =1+\frac{\gamma_{j} /\left(2 \pi^{2}\right)}{\beta_{j}+\gamma_{j}\left(a_{j}^{2}-a_{j}+\frac{1}{3}\right)} \frac{\pi^{2}}{6} \\
& =1+\frac{\gamma_{j}}{12\left(\beta_{j}+\gamma_{j}\left(a_{j}^{2}-a_{j}+\frac{1}{3}\right)\right)},
\end{aligned}
$$


then the mean of $e_{n, d}^{2}\left(P_{n, d}(\boldsymbol{z}), K_{d}^{*}\right)$ over all $\boldsymbol{z} \in \mathbb{Z}_{n}^{d}$ satisfies

$$
\frac{1}{(n-1)^{d}} \sum_{\boldsymbol{z} \in \mathbb{Z}_{n}^{d}} e_{n, d}^{2}\left(P_{n, d}(\boldsymbol{z}), K_{d}^{*}\right) \leq \frac{1}{n} \prod_{j=1}^{d}\left(\hat{\beta}_{j}+2 \hat{\gamma}_{j} \zeta(2)\right) .
$$

Upon making the substitutions $\hat{\beta}_{j}=\beta_{j}+\gamma_{j}\left(a_{j}^{2}-a_{j}+\frac{1}{3}\right)$ and $\hat{\gamma}_{j}=\frac{\gamma_{j}}{2 \pi^{2}}$, and then simplifying, we obtain

$$
\begin{aligned}
\frac{1}{(n-1)^{d}} \sum_{\boldsymbol{z} \in \mathbb{Z}_{n}^{d}} e_{n, d}^{2}\left(P_{n, d}(\boldsymbol{z}), K_{d}^{*}\right) & \leq \frac{1}{n} \prod_{j=1}^{d}\left(\beta_{j}+\gamma_{j}\left(a_{j}^{2}-a_{j}+\frac{1}{2}\right)\right) \\
& \leq \frac{1}{n} \prod_{j=1}^{d}\left(\beta_{j}+\frac{\gamma_{j}}{2}\right) .
\end{aligned}
$$

Hence there exists a choice of $\boldsymbol{z} \in \mathbb{Z}_{n}^{d}$ such that

$$
e_{n, d}^{2}\left(P_{n, d}(\boldsymbol{z}), K_{d}^{*}\right) \leq \frac{1}{n} \prod_{j=1}^{d}\left(\beta_{j}+\frac{\gamma_{j}}{2}\right)
$$

In turn there exists a choice of $\boldsymbol{\Delta} \in[0,1)^{d}$ such that

$$
e_{n, d}^{2}\left(P_{n, d}(\boldsymbol{z}, \boldsymbol{\Delta}), K_{d}\right) \leq e_{n, d}^{2}\left(P_{n, d}(\boldsymbol{z}), K_{d}^{*}\right) \leq \frac{1}{n} \prod_{j=1}^{d}\left(\beta_{j}+\frac{\gamma_{j}}{2}\right) .
$$

Since $a^{2}-a+\frac{1}{3}=\left(a-\frac{1}{2}\right)^{2}+\frac{1}{12} \geq \frac{1}{12}$, a sufficient condition for (4.1) to hold is that, for each $j \geq 1$,

$$
n \geq 1+\frac{\gamma_{j}}{12 \beta_{j}+\gamma_{j}}
$$

Since $\beta_{j}, \gamma_{j}>0$ for each $j$, this condition holds when $n \geq 2$, and so is automatically true when $n$ is a prime number. This completes the proof.

For simplicity, we will take $\boldsymbol{a}=\mathbf{1}$ in the rest of the paper. The remaining theorems can be extended to a general $\boldsymbol{a}$, but the resulting proofs are more involved. When $\boldsymbol{a}=\mathbf{1}$, it follows from Lemma 2.3 that, for $K_{d}=K_{d, \boldsymbol{\beta}, \boldsymbol{\gamma}, \mathbf{1}}$ and any choice of $P_{n, d}=\left\{\boldsymbol{x}_{0}, \ldots, \boldsymbol{x}_{n-1}\right\}$,

$$
\begin{aligned}
e_{n, d}^{2}\left(P_{n, d}, K_{d}\right)= & \prod_{j=1}^{d}\left(\beta_{j}+\frac{\gamma_{j}}{3}\right)-\frac{2}{n} \sum_{i=0}^{n-1} \prod_{j=1}^{d}\left[\beta_{j}+\frac{\gamma_{j}}{2}\left(1-x_{i, j}^{2}\right)\right] \\
& +\frac{1}{n^{2}} \sum_{i=0}^{n-1} \sum_{k=0}^{n-1} \prod_{j=1}^{d}\left[\beta_{j}+\gamma_{j}\left(1-\max \left(x_{i, j}, x_{k, j}\right)\right)\right] .
\end{aligned}
$$

Now suppose that for some particular $d$ we happen to know a set $P_{n, d}$ of $d$ dimensional points $\left\{\boldsymbol{x}_{0}, \ldots, \boldsymbol{x}_{n-1}\right\} \subseteq[0,1)^{d}$ (which may or may not be the points of a shifted lattice rule), for which the bound in Theorem 4.1 is satisfied. Then Theorem 4.4 below will show that it is possible to construct a $(d+1)$-dimensional rule which satisfies the same bound as in Theorem 4.1 but with $d$ replaced by $d+1$. 
The $(d+1)$-dimensional rule uses the set of points

$$
P_{n, d+1}\left(P_{n, d}, z_{d+1}, \Delta_{d+1}\right):=\left\{\left(\boldsymbol{x}_{i},\left\{\frac{i z_{d+1}}{n}+\Delta_{d+1}\right\}\right): i=0,1, \ldots, n-1\right\}
$$

where $z_{d+1} \in \mathbb{Z}_{n}$ and $\Delta_{d+1} \in[0,1)$, so that the first $d$ components of the points in the set $P_{n, d+1}\left(P_{n, d}, z_{d+1}, \Delta_{d+1}\right)$ are exactly the points of $P_{n, d}$. If $P_{n, 1}$ is a 1-dimensional lattice rule, then the $(d+1)$-dimensional rule constructed recursively in this way is a shifted lattice rule.

Theorem 4.4 makes essential use of the quantity

$$
M_{n, d+1}^{2}\left(P_{n, d}, z_{d+1}\right):=\int_{0}^{1} e_{n, d+1}^{2}\left(P_{n, d+1}\left(P_{n, d}, z_{d+1}, \Delta_{d+1}\right), K_{d+1}\right) \mathrm{d} \Delta_{d+1},
$$

which is the mean of the square worst-case error for the $(d+1)$-dimensional rule with points $P_{n, d+1}\left(P_{n, d}, z_{d+1}, \Delta_{d+1}\right)$ taken over all values of the shift $\Delta_{d+1}$.

Lemma 4.2. Let the set $P_{n, d}=\left\{\boldsymbol{x}_{0}, \ldots, \boldsymbol{x}_{n-1}\right\} \subseteq[0,1)^{d}$ and the integer $z_{d+1} \in$ $\mathbb{Z}_{n}$ be given. Then

$$
\begin{aligned}
& M_{n, d+1}^{2}\left(P_{n, d}, z_{d+1}\right) \\
&=\prod_{j=1}^{d+1}\left(\beta_{j}+\frac{\gamma_{j}}{3}\right)-\frac{2}{n} \sum_{i=0}^{n-1}\left[\prod_{j=1}^{d}\left(\beta_{j}+\frac{\gamma_{j}}{2}\left(1-x_{i, j}^{2}\right)\right)\left(\beta_{d+1}+\frac{\gamma_{d+1}}{3}\right)\right] \\
&+\frac{1}{n^{2}} \sum_{i=0}^{n-1} \sum_{k=0}^{n-1}\left[\prod_{j=1}^{d}\left(\beta_{j}+\gamma_{j}\left[1-\max \left(x_{i, j}, x_{k, j}\right)\right]\right)\right. \\
&\left.\cdot\left(\beta_{d+1}+\gamma_{d+1}\left[B_{2}\left(\left\{\frac{(i-k) z_{d+1}}{n}\right\}\right)+\frac{1}{3}\right]\right)\right] .
\end{aligned}
$$

Proof. For any $z_{d+1} \in \mathbb{Z}_{n}$ and any $\Delta_{d+1} \in[0,1)$, suppose we define $W_{i}\left(\Delta_{d+1}\right):=$ $\left\{\frac{i z_{d+1}}{n}+\Delta_{d+1}\right\}$. We have from (4.2) that

$$
\begin{aligned}
e_{n, d+1}^{2}( & \left.P_{n, d+1}\left(P_{n, d}, z_{d+1}, \Delta_{d+1}\right), K_{d+1}\right) \\
= & \prod_{j=1}^{d+1}\left(\beta_{j}+\frac{\gamma_{j}}{3}\right) \\
& -\frac{2}{n} \sum_{i=0}^{n-1}\left[\prod_{j=1}^{d}\left(\beta_{j}+\frac{\gamma_{j}}{2}\left(1-x_{i, j}^{2}\right)\right)\left(\beta_{d+1}+\frac{\gamma_{d+1}}{2}\left(1-W_{i}^{2}\left(\Delta_{d+1}\right)\right)\right)\right] \\
& +\frac{1}{n^{2}} \sum_{i=0}^{n-1} \sum_{k=0}^{n-1}\left[\prod_{j=1}^{d}\left(\beta_{j}+\gamma_{j}\left[1-\max \left(x_{i, j}, x_{k, j}\right)\right]\right)\right. \\
& \left.\cdot\left(\beta_{d+1}+\gamma_{d+1}\left[1-\max \left(W_{i}\left(\Delta_{d+1}\right), W_{j}\left(\Delta_{d+1}\right)\right)\right]\right)\right] .
\end{aligned}
$$


Thus the mean square worst-case error defined above is

$$
\begin{aligned}
& M_{n, d+1}^{2}\left(P_{n, d}, z_{d+1}\right) \\
&=\prod_{j=1}^{d+1}\left(\beta_{j}+\frac{\gamma_{j}}{3}\right)-\frac{2}{n} \sum_{i=0}^{n-1}\left[\prod_{j=1}^{d}\left(\beta_{j}+\frac{\gamma_{j}}{2}\left(1-x_{i, j}^{2}\right)\right)\right. \\
&\left.\cdot\left(\beta_{d+1}+\frac{\gamma_{d+1}}{2}\left(1-\int_{0}^{1} W_{i}^{2}\left(\Delta_{d+1}\right) \mathrm{d} \Delta_{d+1}\right)\right)\right] \\
&+\frac{1}{n^{2}} \sum_{i=0}^{n-1} \sum_{k=0}^{n-1}\left[\prod_{j=1}^{d}\left(\beta_{j}+\gamma_{j}\left[1-\max \left(x_{i, j}, x_{k, j}\right)\right]\right)\right. \\
&\left.\cdot\left(\beta_{d+1}+\gamma_{d+1}\left[1-\int_{0}^{1} \max \left(W_{i}\left(\Delta_{d+1}\right), W_{k}\left(\Delta_{d+1}\right)\right) \mathrm{d} \Delta_{d+1}\right]\right)\right] .
\end{aligned}
$$

For any $x, y \in \mathbb{R}$, it may be verified that

$$
\int_{0}^{1}\{x+\Delta\}^{2} \mathrm{~d} \Delta=\frac{1}{3}
$$

and

$$
\begin{aligned}
\int_{0}^{1} \max (\{x+\Delta\},\{y+\Delta\}) \mathrm{d} \Delta & =\frac{1}{2}-|\{x\}-\{y\}|^{2}+|\{x\}-\{y\}| \\
& =\frac{2}{3}-B_{2}(|\{x\}-\{y\}|) .
\end{aligned}
$$

Since $W_{i}\left(\Delta_{d+1}\right)=\left\{\frac{i z_{d+1}}{n}+\Delta_{d+1}\right\}$ and

$$
B_{2}\left(\left|\left\{\frac{i z_{d+1}}{n}\right\}-\left\{\frac{k z_{d+1}}{n}\right\}\right|\right)=B_{2}\left(\left\{\frac{(i-k) z_{d+1}}{n}\right\}\right),
$$

the result follows.

We shall find it convenient to introduce also a discrete form of the mean (4.3),

$$
\widetilde{M}_{n, d+1}^{2}\left(P_{n, d}, z_{d+1}\right):=\frac{1}{n} \sum_{m=1}^{n} e_{n, d+1}^{2}\left(P_{n, d+1}\left(P_{n, d}, z_{d+1}, \frac{2 m-1}{2 n}\right), K_{d+1}\right),
$$

which is just the composite mid-point rule with spacing $1 / n$ applied to the integral in 4.3.

Lemma 4.3. Let the set $P_{n, d}=\left\{\boldsymbol{x}_{0}, \ldots, \boldsymbol{x}_{n-1}\right\} \subseteq[0,1)^{d}$ and the integer $z_{d+1} \in$ $\mathbb{Z}_{n}$ be given. Then

$$
\widetilde{M}_{n, d+1}^{2}\left(P_{n, d}, z_{d+1}\right) \leq M_{n, d+1}^{2}\left(P_{n, d}, z_{d+1}\right) .
$$

Proof. For any $x, y \in \mathbb{R}$, we have

$$
\begin{aligned}
\int_{0}^{1} \max (\{x+\Delta\},\{y+\Delta\}) \mathrm{d} \Delta \\
=\sum_{m=1}^{n} \int_{\frac{m-1}{n}}^{\frac{m}{n}} \max (\{x+\Delta\},\{y+\Delta\}) \mathrm{d} \Delta
\end{aligned}
$$


and

$$
\int_{0}^{1}\{x+\Delta\}^{2} \mathrm{~d} \Delta=\sum_{m=1}^{n} \int_{\frac{m-1}{n}}^{\frac{m}{n}}\{x+\Delta\}^{2} \mathrm{~d} \Delta .
$$

These two integrals appear in (4.4) with $x$ and $y$ being multiples of $1 / n$. As a result, the values $\max (\{x+\Delta\},\{y+\Delta\})$ and $\{x+\Delta\}^{2}$ are continuous and differentiable on each subinterval $[(m-1) / n, m / n]$ of length $1 / n$. Moreover, $\max (\{x+\Delta\},\{y+\Delta\})$ is linear on each subinterval and so the result of applying the midpoint rule on each subinterval is exact. Thus

$$
\begin{aligned}
& \int_{0}^{1} \max (\{x+\Delta\},\{y+\Delta\}) \mathrm{d} \Delta \\
& \quad=\frac{1}{n} \sum_{m=1}^{n} \max \left(\left\{x+\frac{2 m-1}{2 n}\right\},\left\{y+\frac{2 m-1}{2 n}\right\}\right) .
\end{aligned}
$$

On the other hand, $\{x+\Delta\}^{2}$ is quadratic with a positive second derivative on each subinterval, and so, upon applying the mid-point rule, we have, for $m=1, \ldots, n$,

$$
\int_{\frac{m-1}{n}}^{\frac{m}{n}}\{x+\Delta\}^{2} \mathrm{~d} \Delta>\frac{1}{n}\left\{x+\frac{2 m-1}{2 n}\right\}^{2} .
$$

Hence

$$
\int_{0}^{1}\{x+\Delta\}^{2} \mathrm{~d} \Delta>\frac{1}{n} \sum_{m=1}^{n}\left\{x+\frac{2 m-1}{2 n}\right\}^{2} .
$$

Since $\widetilde{M}_{n, d+1}^{2}\left(P_{n, d}, z_{d+1}\right)$ is just the approximation to $M_{n, d+1}^{2}\left(P_{n, d}, z_{d+1}\right)$ in (4.4) obtained by the composite mid-point rule with spacing $1 / n$, the result follows.

Theorem 4.4. Let $n$ be a prime number and let the set

$$
P_{n, d}=\left\{\boldsymbol{x}_{0}, \ldots, \boldsymbol{x}_{n-1}\right\} \subseteq[0,1)^{d}
$$

be given. Suppose that

$$
e_{n, d}^{2}\left(P_{n, d}, K_{d}\right) \leq \frac{1}{n} \prod_{j=1}^{d}\left(\beta_{j}+\gamma_{j}\right)
$$

Then there exist $z_{d+1} \in \mathbb{Z}_{n}$ and $\Delta_{d+1} \in[0,1)$ such that

$$
e_{n, d+1}^{2}\left(P_{n, d+1}\left(P_{n, d}, z_{d+1}, \Delta_{d+1}\right), K_{d+1}\right) \leq \frac{1}{n} \prod_{j=1}^{d+1}\left(\beta_{j}+\gamma_{j}\right) .
$$

A pair $\left(z_{d+1}, \Delta_{d+1}\right)$ that achieves this bound can be found by first finding a $z_{d+1}$ that minimizes $M_{n, d+1}^{2}\left(P_{n, d}, z_{d+1}\right)$ in Lemma 4.2 and then (with $z_{d+1}$ fixed) finding $a \Delta_{d+1}$ that satisfies (4.7). In the last step it is sufficient to restrict the search over $\Delta_{d+1}$ to the set $\{(2 m-1) /(2 n): m=1, \ldots, n\}$. 
Proof. For any $z_{d+1} \in \mathbb{Z}_{n}$, it follows from Lemma 4.2 and (4.2) that

$$
\begin{aligned}
M_{n, d+1}^{2}\left(P_{n, d}, z_{d+1}\right)=\left(\beta_{d+1}+\frac{\gamma_{d+1}}{3}\right) e_{n, d}^{2}\left(P_{n, d}, K_{d}\right) \\
+\frac{\gamma_{d+1}}{n^{2}} \sum_{i=0}^{n-1} \sum_{k=0}^{n-1}\left[\prod_{j=1}^{d}\left(\beta_{j}+\gamma_{j}\left[1-\max \left(x_{i, j}, x_{k, j}\right)\right]\right)\right. \\
\left.\cdot B_{2}\left(\left\{\frac{(i-k) z_{d+1}}{n}\right\}\right)\right]
\end{aligned}
$$

On separating the $i=k$ terms and the $i \neq k$ terms, we obtain

$$
\begin{aligned}
M_{n, d+1}^{2}( & \left.P_{n, d}, z_{d+1}\right) \\
= & \left(\beta_{d+1}+\frac{\gamma_{d+1}}{3}\right) e_{n, d}^{2}\left(P_{n, d}, K_{d}\right) \\
& +\frac{\gamma_{d+1}}{n^{2}} \sum_{i=0}^{n-1}\left[\prod_{j=1}^{d}\left(\beta_{j}+\gamma_{j}\left(1-x_{i, j}\right)\right) B_{2}(0)\right] \\
& +\frac{\gamma_{d+1}}{n^{2}} \sum_{i=0}^{n-1} \sum_{\substack{k=0 \\
k \neq i}}^{n-1}\left[\prod_{j=1}^{d}\left(\beta_{j}+\gamma_{j}\left[1-\max \left(x_{i, j}, x_{i, k}\right)\right]\right)\right. \\
= & \left(\beta_{d+1}+\frac{\gamma_{d+1}}{3}\right) e_{n, d}^{2}\left(P_{n, d}, K_{d}\right)+\frac{\gamma_{d+1}}{6 n^{2}} \sum_{i=0}^{n-1} \prod_{j=1}^{d}\left(\beta_{j}+\gamma_{j}\left(1-x_{i, j}\right)\right) \\
& +\frac{\gamma_{d+1}}{n^{2}} \sum_{i=0}^{n-1} \sum_{\substack{n=0 \\
k \neq i}}^{n-1}\left[\prod_{j=1}^{d}\left(\beta_{j}+\gamma_{j}\left[1-\max \left(x_{i, j}, x_{k, j}\right)\right]\right)\right. \\
& \left.\cdot\left(\frac{1}{2 \pi^{2}} \sum_{h=-\infty}^{\infty} \frac{e^{2 \pi \mathrm{i} h(i-k) z_{d+1} / n}}{h^{2}}\right)\right]
\end{aligned}
$$

where we have used (3.2). Now we average this over all possible values of $z_{d+1} \in \mathbb{Z}_{n}$, forming

$$
\begin{aligned}
\Psi_{n, d+1}\left(P_{n, d}\right):= & \frac{1}{n-1} \sum_{z_{d+1}=1}^{n-1} M_{n, d+1}^{2}\left(P_{n, d}, z_{d+1}\right) \\
= & \left(\beta_{d+1}+\frac{\gamma_{d+1}}{3}\right) e_{n, d}^{2}\left(P_{n, d}, K_{d}\right) \\
& +\frac{\gamma_{d+1}}{6 n^{2}} \sum_{i=0}^{n-1} \prod_{j=1}^{d}\left[\beta_{j}+\gamma_{j}\left(1-x_{i, j}\right)\right]+\psi_{n, d+1},
\end{aligned}
$$


where

$$
\begin{array}{r}
\psi_{n, d+1}:=\frac{1}{n-1} \sum_{z_{d+1}=1}^{n-1}\left[\frac { \gamma _ { d + 1 } } { n ^ { 2 } } \sum _ { i = 0 } ^ { n - 1 } \sum _ { \substack { k = 0 \\
k \neq i } } ^ { n - 1 } \left[\prod_{j=1}^{d}\left(\beta_{j}+\gamma_{j}\left[1-\max \left(x_{i, j}, x_{k, j}\right)\right]\right)\right.\right. \\
\left.\left.\cdot\left(\frac{1}{2 \pi^{2}} \sum_{h=-\infty}^{\infty} \frac{e^{2 \pi \mathrm{i} h(i-k) z_{d+1} / n}}{h^{2}}\right)\right]\right] \\
=\frac{\gamma_{d+1}}{n^{2}} \sum_{i=0}^{n-1} \sum_{\substack{k=0 \\
k \neq i}}^{n-1}\left[\prod_{j=1}^{d}\left(\beta_{j}+\gamma_{j}\left[1-\max \left(x_{i, j}, x_{k, j}\right)\right]\right)\right. \\
\left.\cdot\left(\frac{1}{2 \pi^{2}} \sum_{h=-\infty}^{\infty} \frac{1}{h^{2}}\left(\frac{1}{n-1} \sum_{z_{d+1}=1}^{n-1} e^{2 \pi \mathrm{i} h(i-k) z_{d+1} / n}\right)\right)\right] .
\end{array}
$$

If $h$ is a multiple of $n$, then

$$
\frac{1}{n-1} \sum_{z_{d+1}=1}^{n-1} e^{2 \pi \mathrm{i} h(i-k) z_{d+1} / n}=1 .
$$

If $h$ is not a multiple of $n$, then, noting that $i-k$ is not a multiple of $n$ either, we have

$$
\begin{aligned}
\frac{1}{n-1} \sum_{z_{d+1}=1}^{n-1} e^{2 \pi \mathrm{i} h(i-k) z_{d+1} / n} & =\frac{1}{n-1}\left(\sum_{z_{d+1}=0}^{n-1}\left(e^{2 \pi \mathrm{i} h(i-k) / n}\right)^{z_{d+1}}-1\right) \\
& =-\frac{1}{n-1} .
\end{aligned}
$$

Thus

$$
\begin{aligned}
\frac{1}{2 \pi^{2}} & \sum_{h=-\infty}^{\infty} \frac{1}{h^{2}}\left(\frac{1}{n-1} \sum_{z_{d+1}=1}^{n-1} e^{2 \pi \mathrm{i} h(i-k) z_{d+1} / n}\right) \\
& =\frac{1}{2 \pi^{2}}\left(\sum_{h \equiv 0(\bmod n)}^{\prime} \frac{1}{h^{2}}-\frac{1}{n-1} \sum_{h \neq 0(\bmod n)}^{\prime} \frac{1}{h^{2}}\right) \\
& =\frac{1}{2 \pi^{2}}\left(\sum_{m=-\infty}^{\prime} \frac{1}{(m n)^{2}}-\frac{1}{n-1}\left(\sum_{h=-\infty}^{\infty} \frac{1}{h^{2}}-\sum_{m=-\infty}^{\prime} \frac{1}{(m n)^{2}}\right)\right) \\
& =\frac{1}{2 \pi^{2}}\left(\frac{2 \zeta(2)}{n^{2}}-\frac{1}{n-1}\left(2 \zeta(2)-\frac{2 \zeta(2)}{n^{2}}\right)\right) \\
& =\frac{1}{2 \pi^{2}}\left(-\frac{2 \zeta(2)}{n}\right) \\
& =-\frac{1}{6 n} .
\end{aligned}
$$


It follows that $\psi_{n, d+1}<0$. Hence

$$
\begin{aligned}
\Psi_{n, d+1}\left(P_{n, d}\right) \leq & \left(\beta_{d+1}+\frac{\gamma_{d+1}}{3}\right) e_{n, d}^{2}\left(P_{n, d}, K_{d}\right) \\
& +\frac{\gamma_{d+1}}{6 n^{2}} \sum_{i=0}^{n-1} \prod_{j=1}^{d}\left[\beta_{j}+\gamma_{j}\left(1-x_{i, j}\right)\right] \\
\leq & \left(\beta_{d+1}+\frac{\gamma_{d+1}}{3}\right) \frac{1}{n} \prod_{j=1}^{d}\left(\beta_{j}+\gamma_{j}\right)+\frac{\gamma_{d+1}}{6 n} \prod_{j=1}^{d}\left(\beta_{j}+\gamma_{j}\right) \\
= & \left(\beta_{d+1}+\frac{\gamma_{d+1}}{2}\right) \frac{1}{n} \prod_{j=1}^{d}\left(\beta_{j}+\gamma_{j}\right) \\
\leq & \frac{1}{n} \prod_{j=1}^{d+1}\left(\beta_{j}+\gamma_{j}\right) .
\end{aligned}
$$

Since $\Psi_{n, d+1}\left(P_{n, d}\right)$ is the average of $M_{n, d+1}^{2}\left(P_{n, d}, z_{d+1}\right)$ over all $z_{d+1}$, if we choose $z_{d+1} \in \mathbb{Z}_{n}$ to minimize $M_{n, d+1}^{2}\left(P_{n, d}, z_{d+1}\right)$, then this choice of $z_{d+1}$ will satisfy

$$
M_{n, d+1}^{2}\left(P_{n, d}, z_{d+1}\right) \leq \Psi_{n, d+1}\left(P_{n, d}\right) \leq \frac{1}{n} \prod_{j=1}^{d+1}\left(\beta_{j}+\gamma_{j}\right) .
$$

Now for this particular choice of $z_{d+1}, \widetilde{M}_{n, d+1}^{2}\left(P_{n, d}, z_{d+1}\right)$ is the average of

$$
e_{n, d+1}^{2}\left(P_{n, d+1}\left(P_{n, d}, z_{d+1}, \Delta_{d+1}\right), K_{d+1}\right)
$$

over all $\Delta_{d+1}$ in the set $\{(2 m-1) /(2 n): m=1, \ldots, n\}$. Therefore if we choose $\Delta_{d+1}$ from this finite set to minimize $e_{n, d+1}^{2}\left(P_{n, d+1}\left(P_{n, d}, z_{d+1}, \Delta_{d+1}\right), K_{d+1}\right)$, then this choice of $\Delta_{d+1}$ will satisfy

$$
e_{n, d+1}^{2}\left(P_{n, d+1}\left(P_{n, d}, z_{d+1}, \Delta_{d+1}\right), K_{d+1}\right) \leq \widetilde{M}_{n, d+1}^{2}\left(P_{n, d}, z_{d+1}\right),
$$

and in turn it follows from Lemma 4.3 that

$$
e_{n, d+1}^{2}\left(P_{n, d+1}\left(P_{n, d}, z_{d+1}, \Delta_{d+1}\right), K_{d+1}\right) \leq \frac{1}{n} \prod_{j=1}^{d+1}\left(\beta_{j}+\gamma_{j}\right) .
$$

This completes the proof.

\section{The COMPONENT-BY-COMPONENT SEARCH ALGORITHM}

We now introduce the simpler notation

$$
e_{n, d}(\boldsymbol{z}, \boldsymbol{\Delta}):=e_{n, d}\left(P_{n, d}(\boldsymbol{z}, \boldsymbol{\Delta}), K_{d, \beta, \gamma, \mathbf{1}}\right) .
$$

Given $n$ (a prime number) and $d$, Theorem 4.4 leads to the following algorithm for finding a generator vector $\boldsymbol{z}=\left(z_{1}, z_{2}, \ldots, z_{d}\right)$ and a shift $\boldsymbol{\Delta}=\left(\Delta_{1}, \Delta_{2}, \ldots, \Delta_{d}\right)$ such that

$$
e_{n, \ell}^{2}\left(\left(z_{1}, z_{2}, \ldots, z_{\ell}\right),\left(\Delta_{1}, \Delta_{2}, \ldots, \Delta_{\ell}\right)\right) \leq \frac{1}{n} \prod_{j=1}^{\ell}\left(\beta_{j}+\gamma_{j}\right),
$$

for all $\ell=1, \ldots, d$.

If at a later time further dimensions are needed, the algorithm can be restarted in an obvious way. 


\section{Algorithm 5.1.}

1. Set $z_{1}$, the first component of $\boldsymbol{z}$, equal to 1 .

2. Find $\Delta_{1} \in\{1 /(2 n), 3 /(2 n), \ldots,(2 n-1) /(2 n)\}$ to minimize

$$
\begin{aligned}
e_{n, 1}^{2}\left(z_{1}, \Delta_{1}\right) & \\
= & \left(\beta_{1}+\frac{\gamma_{1}}{3}\right)-\frac{2}{n} \sum_{i=0}^{n-1}\left[\beta_{1}+\frac{\gamma_{1}}{2}\left(1-\left\{\frac{i z_{1}}{n}+\Delta_{1}\right\}^{2}\right)\right] \\
& +\frac{1}{n^{2}} \sum_{i=0}^{n-1} \sum_{k=0}^{n-1}\left[\beta_{1}+\gamma_{1}\left(1-\max \left(\left\{\frac{i z_{1}}{n}+\Delta_{1}\right\},\left\{\frac{k z_{1}}{n}+\Delta_{1}\right\}\right)\right)\right] .
\end{aligned}
$$

3. For $\ell=2,3, \ldots, d-1, d$, do the following:

(a) Find $z_{\ell} \in \mathbb{Z}_{n}=\{1,2, \ldots, n-1\}$ to minimize

$$
\begin{aligned}
& M_{n, \ell}^{2}\left(\left(z_{1}, z_{2}, \ldots, z_{\ell-1}\right),\left(\Delta_{1}, \Delta_{2}, \ldots, \Delta_{\ell-1}\right), z_{\ell}\right) \\
& =\left(\beta_{\ell}+\frac{\gamma_{\ell}}{3}\right) e_{n, \ell-1}^{2}\left(\left(z_{1}, z_{2}, \ldots, z_{\ell-1}\right),\left(\Delta_{1}, \Delta_{2}, \ldots, \Delta_{\ell-1}\right)\right) \\
& +\frac{\gamma_{\ell}}{n^{2}} \sum_{i=0}^{n-1} \sum_{k=0}^{n-1}\left[\prod_{j=1}^{\ell-1}\left(\beta_{j}+\gamma_{j}\left[1-\max \left(\left\{\frac{i z_{j}}{n}+\Delta_{j}\right\},\left\{\frac{k z_{j}}{n}+\Delta_{j}\right\}\right)\right]\right)\right. \\
& \left.\cdot B_{2}\left(\left\{\frac{(i-k) z_{\ell}}{n}\right\}\right)\right] .
\end{aligned}
$$

(b) Find $\Delta_{\ell} \in\{1 /(2 n), 3 /(2 n), \ldots,(2 n-1) /(2 n)\}$ to minimize

$$
\begin{aligned}
e_{n, \ell}^{2}\left(\left(z_{1}, z_{2}, \ldots, z_{\ell}\right),\left(\Delta_{1}, \Delta_{2}, \ldots, \Delta_{\ell}\right)\right) \\
=\prod_{j=1}^{\ell}\left(\beta_{j}+\frac{\gamma_{j}}{3}\right)-\frac{2}{n} \sum_{i=0}^{n-1} \prod_{j=1}^{\ell}\left[\beta_{j}+\frac{\gamma_{j}}{2}\left(1-\left\{\frac{i z_{j}}{n}+\Delta_{j}\right\}^{2}\right)\right] \\
\quad+\frac{1}{n^{2}} \sum_{i=0}^{n-1} \sum_{k=0}^{n-1} \prod_{j=1}^{\ell}\left[\beta_{j}+\gamma_{j}\left(1-\max \left(\left\{\frac{i z_{j}}{n}+\Delta_{j}\right\},\left\{\frac{k z_{j}}{n}+\Delta_{j}\right\}\right)\right)\right] .
\end{aligned}
$$

We see from the algorithm that the cost of constructing a rule for all dimensions up to $d$ is $O\left(n^{3} d^{2}\right)$. This can be reduced to $O\left(n^{3} d\right)$ if we store the products during the search. However, this would require $O\left(n^{2}\right)$ storage.

The next theorem states, in effect, that the QMC rules found by the present procedure conform with the strong QMC tractability bound of 8 . At the same time it confirms that the $\varepsilon$-exponent (see the Introduction) is at most 2.

Theorem 5.2. Let $n$ be a prime number, and let $\boldsymbol{z} \in \mathbb{Z}_{n}^{d}$ and $\boldsymbol{\Delta} \in[0,1)^{d}$ be obtained from Algorithm [5.1] Suppose that

$$
\sum_{j=1}^{\infty} \frac{\gamma_{j}}{\beta_{j}}<\infty .
$$

Then for all $d \geq 1$ we have

$$
e_{n, d}(\boldsymbol{z}, \boldsymbol{\Delta}) \leq C n^{-\frac{1}{2}} e_{0, d},
$$


with

$$
C=\prod_{j=1}^{\infty}\left(1+\frac{2 \gamma_{j}}{3 \beta_{j}+\gamma_{j}}\right)^{\frac{1}{2}} \leq \exp \left(\frac{1}{3} \sum_{j=1}^{\infty} \frac{\gamma_{j}}{\beta_{j}}\right)<\infty .
$$

Proof. Because $\Delta_{1}$ is chosen from $\{1 /(2 n), 3 /(2 n), \ldots,(2 n-1) /(2 n)\}$ by minimizing $e_{n, 1}^{2}\left(1, \Delta_{1}\right)$ (see Step 2 of Algorithm 5.1 ), it follows that

$$
e_{n, 1}^{2}\left(1, \Delta_{1}\right) \leq \frac{1}{n} \sum_{m=1}^{n} e_{n, 1}^{2}\left(1, \frac{2 m-1}{2 n}\right)
$$

Arguments similar to those used in the proof of Lemma 4.3 show that

$$
\frac{1}{n} \sum_{m=1}^{n} e_{n, 1}^{2}\left(1, \frac{2 m-1}{2 n}\right) \leq \int_{0}^{1} e_{n, 1}^{2}(1, \Delta) \mathrm{d} \Delta .
$$

By using (4.5), 4.6), and $B_{2}(x)=x^{2}-x+1 / 6$, we can work out this integral to obtain

$$
\int_{0}^{1} e_{n, 1}^{2}(1, \Delta) \mathrm{d} \Delta=\frac{\gamma_{1}}{6 n^{2}}<\frac{1}{n}\left(\beta_{1}+\gamma_{1}\right)
$$

Hence $e_{n, 1}^{2}\left(1, \Delta_{1}\right) \leq\left(\beta_{1}+\gamma_{1}\right) / n$. Then Theorem 4.4 shows that the vectors $\boldsymbol{z} \in \mathbb{Z}_{n}^{d}$ and $\boldsymbol{\Delta} \in[0,1)^{d}$ found using Algorithm 5.1 satisfy

$$
e_{n, d}^{2}(\boldsymbol{z}, \boldsymbol{\Delta}) \leq \frac{1}{n} \prod_{j=1}^{d}\left(\beta_{j}+\gamma_{j}\right)=\frac{1}{n}\left(\prod_{j=1}^{d} \frac{\beta_{j}+\gamma_{j}}{\beta_{j}+\frac{\gamma_{j}}{3}}\right)\left(\prod_{j=1}^{d}\left(\beta_{j}+\frac{\gamma_{j}}{3}\right)\right) .
$$

Now recall that the initial worst-case error $e_{0, d}$ is given by $\left\|h_{d}\right\|_{d}$, which from (2.6) with $a_{j}=1$ is

$$
e_{0, d}=\prod_{j=1}^{d}\left(\beta_{j}+\frac{\gamma_{j}}{3}\right)^{\frac{1}{2}}
$$

Thus

$$
\begin{aligned}
e_{n, d}^{2}(\boldsymbol{z}, \boldsymbol{\Delta}) & \leq \frac{1}{n} \prod_{j=1}^{d}\left(1+\frac{2 \gamma_{j}}{3 \beta_{j}+\gamma_{j}}\right) e_{0, d}^{2} \\
& \leq \frac{1}{n} \prod_{j=1}^{\infty}\left(1+\frac{2 \gamma_{j}}{3 \beta_{j}+\gamma_{j}}\right) e_{0, d}^{2} .
\end{aligned}
$$

It then follows that $e_{n, d}(\boldsymbol{z}, \boldsymbol{\Delta}) \leq C n^{-\frac{1}{2}} e_{0, d}$, where

$$
\begin{aligned}
C & =\prod_{j=1}^{\infty}\left(1+\frac{2 \gamma_{j}}{3 \beta_{j}+\gamma_{j}}\right)^{\frac{1}{2}} \leq \prod_{j=1}^{\infty}\left(1+\frac{2 \gamma_{j}}{3 \beta_{j}}\right)^{\frac{1}{2}} \\
& =\exp \left(\frac{1}{2} \sum_{j=1}^{\infty} \log \left(1+\frac{2 \gamma_{j}}{3 \beta_{j}}\right)\right) \\
& \leq \exp \left(\frac{1}{2} \sum_{j=1}^{\infty} \frac{2 \gamma_{j}}{3 \beta_{j}}\right)=\exp \left(\frac{1}{3} \sum_{j=1}^{\infty} \frac{\gamma_{j}}{\beta_{j}}\right)
\end{aligned}
$$


where in the penultimate step we have used the fact that $\log (1+x) \leq x$ for all $x \geq 0$. Since $\sum_{j=1}^{\infty} \frac{\gamma_{j}}{\beta_{j}}<\infty, C$ is bounded.

We remark that for fixed $d$, this proof shows that for any choice of positive $\beta_{j}$ and $\gamma_{j}$, we have

$$
e_{n, d}(\boldsymbol{z}, \boldsymbol{\Delta}) \leq \frac{1}{\sqrt{n}} \prod_{j=1}^{d}\left(1+\frac{2 \gamma_{j}}{3 \beta_{j}+\gamma_{j}}\right)^{\frac{1}{2}} e_{0, d}
$$

In particular, for the classical case $\beta_{j}=\gamma_{j}=1$, we obtain

$$
e_{n, d}(\boldsymbol{z}, \boldsymbol{\Delta}) \leq \frac{1}{\sqrt{n}}\left(\frac{3}{2}\right)^{\frac{d}{2}} e_{0, d}
$$

Moreover, if we have

$$
\eta=\limsup _{d \rightarrow \infty} \frac{\sum_{j=1}^{\infty} \frac{\gamma_{j}}{\beta_{j}}}{\log d}<\infty
$$

then arguments similar to those used in the proof of Theorem 3 in 9] show that the QMC rules found by Algorithm 5.1 satisfy a tractability bound that has a polynomial dependence on $d$ of approximately $d^{2 \eta / 3}$.

\section{NumericAl RESUlts OF SEARCHES}

We consider the weighted Sobolev spaces with $\beta_{j}=1$ for each $j$ and four different sequences of $\gamma$ :

$$
\gamma_{j}=\frac{1}{j^{2}}, \quad \gamma_{j}=0.5^{j}, \quad \gamma_{j}=0.75^{j}, \quad \gamma_{j}=0.9^{j}
$$

Some preliminary numerical searches for the generator vector and the shift were carried out using Algorithm 5.1 with $n$ taking the prime values 1009, 2003, and 4001. In the implementation of Step 3(a) of Algorithm 5.1 we only considered values of $z_{\ell}$ in $\left\{1,2, \ldots, \frac{n-1}{2}\right\}$, since

$$
\begin{aligned}
B_{2}\left(\left\{\frac{(i-k) z_{\ell}}{n}\right\}\right) & =B_{2}\left(1-\left\{\frac{(i-k)\left(n-z_{\ell}\right)}{n}\right\}\right) \\
& =B_{2}\left(\left\{\frac{(i-k)\left(n-z_{\ell}\right)}{n}\right\}\right) .
\end{aligned}
$$

We compare our results with the mean square worst-case error over all QMC rules (see Lemma 8 of [8]):

$$
E_{n, d}^{2}:=\frac{1}{n}\left[\prod_{j=1}^{d}\left(\beta_{j}+\frac{\gamma_{j}}{2}\right)-\prod_{j=1}^{d}\left(\beta_{j}+\frac{\gamma_{j}}{3}\right)\right] .
$$

The results are given in the Appendix in tables with entries $d, z_{d}, \Delta_{d}, e_{n, d}(\boldsymbol{z}, \boldsymbol{\Delta})$, and the QMC mean $E_{n, d}$.

The striking feature of the numerical results is that in all cases the worst-case errors $e_{n, d}$ for the computed rules are considerably smaller than the root mean square worst-case errors $E_{n, d}$. It also seems, on comparing the results with $n=$ 1009,2003 and 4001, that the convergence of $e_{n, d}$ to zero as $n$ increases is faster than the theoretically predicted $O\left(n^{-1 / 2}\right)$. 
TABle 1. Maximum ratios of $e_{1009, d}$

\begin{tabular}{|l|c|c|c|c|}
\hline & $\gamma_{j}=1 / j^{2}$ & $\gamma_{j}=0.5^{j}$ & $\gamma_{j}=0.75^{j}$ & $\gamma_{j}=0.9^{j}$ \\
\hline Rules found with $\gamma_{j}=1 / j^{2}$ & 1 & 1.003 & 1.061 & 1.131 \\
Rules found with $\gamma_{j}=0.5^{j}$ & 1.135 & 1 & 1.092 & 1.500 \\
Rules found with $\gamma_{j}=0.75^{j}$ & 1.036 & 1.013 & 1 & 1.037 \\
Rules found with $\gamma_{j}=0.9^{j}$ & 1.083 & 1.024 & 1.028 & 1 \\
\hline
\end{tabular}

Though the results are not presented here, some searches were also done for two other choices of $\gamma_{j}$, namely, $\gamma_{j}=1 / j$ and $\gamma_{j}=1 / j^{1.1}$ with $\beta_{j}=1$ in both cases. For the first choice, we expect tractability, but not strong tractability, whereas strong tractability is expected in the second case. The values of $e_{n, d}(\boldsymbol{z}, \boldsymbol{\Delta})$ for the case $\gamma_{j}=1 / j^{1.1}$ and $d>1$ were smaller than those in the case $\gamma_{j}=1 / j$, which in turn were smaller than the values presented in Tables 5 , 9 and 13 for the case $\gamma_{j}=0.9^{j}$.

In practice, we do not know what the weights for a particular integrand will be. So one may ask the question of whether the shifted lattice rules found using Algorithm 5.1 for a particular choice of the sequence $\gamma$ will be good for other choices of $\gamma$. As a numerical experiment, we took $n=1009$ and used the rules in each of Tables 2 5 (which are for a particular choice of $\gamma$ ) for $d$ up to 40 dimensions to calculate the values of $e_{n, d}(\boldsymbol{z}, \boldsymbol{\Delta})$ for all the other three choices of $\boldsymbol{\gamma}$.

A summary of the results is given in Table 1. To describe what the numerical entries mean, consider the column headed $\gamma_{j}=1 / j^{2}$. Then each entry in the column is

$$
\max _{1 \leq d \leq 40} \frac{e_{1009, d}^{\prime}(\boldsymbol{z}, \boldsymbol{\Delta})}{e_{1009, d}(\boldsymbol{z}, \boldsymbol{\Delta})},
$$

where both $e_{1009, d}^{\prime}(\boldsymbol{z}, \boldsymbol{\Delta})$ and $e_{1009, d}(\boldsymbol{z}, \boldsymbol{\Delta})$ are calculated with $\gamma_{j}=1 / j^{2}$. However, $e_{1009, d}^{\prime}(\boldsymbol{z}, \boldsymbol{\Delta})$ is calculated by using the rule found by applying Algorithm 5.1 and taking the $\gamma_{j}$ given in the corresponding row, whereas $e_{1009, d}(\boldsymbol{z}, \boldsymbol{\Delta})$ is calculated using the rule found by applying Algorithm 5.1 and taking $\gamma_{j}=1 / j^{2}$. Thus for example, in the column with $\gamma_{j}=1 / j^{2}$, we see an entry of 1.083 in the row with $\gamma_{j}=0.9^{j}$. This entry of 1.083 means that for each value of $d$, all the values of $e_{1009, d}^{\prime}(\boldsymbol{z}, \boldsymbol{\Delta})$ calculated with $\gamma_{j}=1 / j^{2}$ using the rules found by applying Algorithm 5.1 with $\gamma_{j}=0.9^{j}$ (see Table 5 for the $z_{d}$ and $\Delta_{d}$ ) were at most $8.3 \%$ larger than the corresponding value of $e_{1009, d}(\boldsymbol{z}, \boldsymbol{\Delta})$ found in Table 2 (corresponding to $\left.\gamma_{j}=1 / j^{2}\right)$. The numerical entries in the other three columns have a similar meaning.

A reasonable conclusion might be that the rules found with $\gamma_{j}=1 / j^{2}$ are attractively robust when used to compute the worst case error with other choices of $\gamma_{j}$.

\section{Discussion}

The theoretical and computational results in this paper show that it is feasible to obtain shifted lattice rules that more than satisfy the strong QMC tractability bounds in weighted Sobolev spaces, for prime values of $n$ up to a few thousand, and dimensions $d$ up to the order of a hundred. Whether such rules will prove useful in practice is yet to be seen. 
Appendix: TABles of Shifted LATtice RUles

TABLE 2. $n=1009$ and $\gamma_{j}=1 / j^{2}$

\begin{tabular}{|r|r|l|l|c|}
\hline$d$ & $z_{d}$ & $\Delta_{d}$ & $e_{n, d}(\boldsymbol{z}, \boldsymbol{\Delta})$ & $E_{n, d}$ \\
\hline 1 & 1 & 0.51883 & $2.8610 \mathrm{e}-04$ & $1.2852 \mathrm{e}-02$ \\
2 & 390 & 0.36620 & $4.5598 \mathrm{e}-04$ & $1.5521 \mathrm{e}-02$ \\
3 & 264 & 0.29386 & $5.6752 \mathrm{e}-04$ & $1.6757 \mathrm{e}-02$ \\
4 & 442 & 0.48612 & $6.4521 \mathrm{e}-04$ & $1.7465 \mathrm{e}-02$ \\
5 & 362 & 0.12735 & $7.0250 \mathrm{e}-04$ & $1.7923 \mathrm{e}-02$ \\
6 & 429 & 0.45243 & $7.4655 \mathrm{e}-04$ & $1.8243 \mathrm{e}-02$ \\
7 & 469 & 0.21457 & $7.8530 \mathrm{e}-04$ & $1.8479 \mathrm{e}-02$ \\
8 & 450 & 0.42270 & $8.1740 \mathrm{e}-04$ & $1.8660 \mathrm{e}-02$ \\
9 & 146 & 0.51189 & $8.4249 \mathrm{e}-04$ & $1.8803 \mathrm{e}-02$ \\
10 & 209 & 0.27304 & $8.6418 \mathrm{e}-04$ & $1.8920 \mathrm{e}-02$ \\
11 & 474 & 0.12735 & $8.8209 \mathrm{e}-04$ & $1.9016 \mathrm{e}-02$ \\
12 & 187 & 0.47621 & $8.9810 \mathrm{e}-04$ & $1.9097 \mathrm{e}-02$ \\
13 & 306 & 0.10159 & $9.1174 \mathrm{e}-04$ & $1.9166 \mathrm{e}-02$ \\
14 & 242 & 0.90337 & $9.2445 \mathrm{e}-04$ & $1.9226 \mathrm{e}-02$ \\
15 & 371 & 0.27502 & $9.3574 \mathrm{e}-04$ & $1.9278 \mathrm{e}-02$ \\
16 & 83 & 0.28989 & $9.4562 \mathrm{e}-04$ & $1.9324 \mathrm{e}-02$ \\
17 & 294 & 0.85481 & $9.5456 \mathrm{e}-04$ & $1.9364 \mathrm{e}-02$ \\
18 & 157 & 0.23241 & $9.6287 \mathrm{e}-04$ & $1.9401 \mathrm{e}-02$ \\
19 & 110 & 0.21853 & $9.7071 \mathrm{e}-04$ & $1.9433 \mathrm{e}-02$ \\
20 & 320 & 0.12834 & $9.7793 \mathrm{e}-04$ & $1.9462 \mathrm{e}-02$ \\
21 & 446 & 0.97572 & $9.8464 \mathrm{e}-04$ & $1.9489 \mathrm{e}-02$ \\
22 & 420 & 0.18186 & $9.9062 \mathrm{e}-04$ & $1.9513 \mathrm{e}-02$ \\
23 & 455 & 0.95788 & $9.9641 \mathrm{e}-04$ & $1.9535 \mathrm{e}-02$ \\
24 & 377 & 0.75768 & $1.0018 \mathrm{e}-03$ & $1.9556 \mathrm{e}-02$ \\
25 & 119 & 0.26511 & $1.0068 \mathrm{e}-03$ & $1.9575 \mathrm{e}-02$ \\
26 & 171 & 0.91923 & $1.0113 \mathrm{e}-03$ & $1.9592 \mathrm{e}-02$ \\
27 & 353 & 0.10951 & $1.0156 \mathrm{e}-03$ & $1.9608 \mathrm{e}-02$ \\
28 & 422 & 0.65956 & $1.0197 \mathrm{e}-03$ & $1.9623 \mathrm{e}-02$ \\
29 & 491 & 0.64767 & $1.0234 \mathrm{e}-03$ & $1.9637 \mathrm{e}-02$ \\
30 & 412 & 0.13231 & $1.0270 \mathrm{e}-03$ & $1.9650 \mathrm{e}-02$ \\
31 & 304 & 0.70614 & $1.0305 \mathrm{e}-03$ & $1.9662 \mathrm{e}-02$ \\
32 & 247 & 0.06293 & $1.0338 \mathrm{e}-03$ & $1.9674 \mathrm{e}-02$ \\
33 & 368 & 0.05600 & $1.0369 \mathrm{e}-03$ & $1.9685 \mathrm{e}-02$ \\
34 & 409 & 0.52379 & $1.0398 \mathrm{e}-03$ & $1.9695 \mathrm{e}-02$ \\
35 & 313 & 0.97671 & $1.0427 \mathrm{e}-03$ & $1.9704 \mathrm{e}-02$ \\
36 & 343 & 0.83102 & $1.0454 \mathrm{e}-03$ & $1.9713 \mathrm{e}-02$ \\
37 & 128 & 0.36918 & $1.0480 \mathrm{e}-03$ & $1.9722 \mathrm{e}-02$ \\
38 & 456 & 0.98464 & $1.0504 \mathrm{e}-03$ & $1.9730 \mathrm{e}-02$ \\
39 & 465 & 0.43162 & $1.0527 \mathrm{e}-03$ & $1.9738 \mathrm{e}-02$ \\
40 & 282 & 0.74579 & $1.0549 \mathrm{e}-03$ & $1.9745 \mathrm{e}-02$ \\
\hline
\end{tabular}


TABLE 3. $n=1009$ and $\gamma_{j}=0.5^{j}$

\begin{tabular}{|r|r|l|l|c|}
\hline$d$ & $z_{d}$ & $\Delta_{d}$ & $e_{n, d}(\boldsymbol{z}, \boldsymbol{\Delta})$ & $E_{n, d}$ \\
\hline 1 & 1 & 0.75173 & $2.0230 \mathrm{e}-04$ & $9.0879 \mathrm{e}-03$ \\
2 & 390 & 0.15411 & $3.3792 \mathrm{e}-04$ & $1.1878 \mathrm{e}-02$ \\
3 & 264 & 0.58920 & $4.3830 \mathrm{e}-04$ & $1.3267 \mathrm{e}-02$ \\
4 & 442 & 0.75471 & $5.0246 \mathrm{e}-04$ & $1.3968 \mathrm{e}-02$ \\
5 & 362 & 0.75372 & $5.4012 \mathrm{e}-04$ & $1.4321 \mathrm{e}-02$ \\
6 & 429 & 0.44549 & $5.6180 \mathrm{e}-04$ & $1.4498 \mathrm{e}-02$ \\
7 & 461 & 0.39693 & $5.7376 \mathrm{e}-04$ & $1.4587 \mathrm{e}-02$ \\
8 & 146 & 0.75669 & $5.7995 \mathrm{e}-04$ & $1.4631 \mathrm{e}-02$ \\
9 & 153 & 0.65461 & $5.8332 \mathrm{e}-04$ & $1.4653 \mathrm{e}-02$ \\
10 & 180 & 0.36521 & $5.8498 \mathrm{e}-04$ & $1.4665 \mathrm{e}-02$ \\
11 & 450 & 0.79732 & $5.8585 \mathrm{e}-04$ & $1.4670 \mathrm{e}-02$ \\
12 & 191 & 0.03320 & $5.8628 \mathrm{e}-04$ & $1.4673 \mathrm{e}-02$ \\
13 & 110 & 0.34737 & $5.8651 \mathrm{e}-04$ & $1.4674 \mathrm{e}-02$ \\
14 & 209 & 0.00842 & $5.8662 \mathrm{e}-04$ & $1.4675 \mathrm{e}-02$ \\
15 & 474 & 0.92815 & $5.8668 \mathrm{e}-04$ & $1.4675 \mathrm{e}-02$ \\
16 & 157 & 0.97968 & $5.8671 \mathrm{e}-04$ & $1.4675 \mathrm{e}-02$ \\
17 & 371 & 0.06690 & $5.8673 \mathrm{e}-04$ & $1.4676 \mathrm{e}-02$ \\
18 & 242 & 0.92121 & $5.8673 \mathrm{e}-04$ & $1.4676 \mathrm{e}-02$ \\
19 & 242 & 0.58722 & $5.8674 \mathrm{e}-04$ & $1.4676 \mathrm{e}-02$ \\
20 & 320 & 0.90436 & $5.8674 \mathrm{e}-04$ & $1.4676 \mathrm{e}-02$ \\
21 & 273 & 0.52775 & $5.8674 \mathrm{e}-04$ & $1.4676 \mathrm{e}-02$ \\
22 & 474 & 0.17493 & $5.8674 \mathrm{e}-04$ & $1.4676 \mathrm{e}-02$ \\
23 & 142 & 0.11645 & $5.8674 \mathrm{e}-04$ & $1.4676 \mathrm{e}-02$ \\
24 & 320 & 0.12537 & $5.8674 \mathrm{e}-04$ & $1.4676 \mathrm{e}-02$ \\
25 & 409 & 0.90238 & $5.8674 \mathrm{e}-04$ & $1.4676 \mathrm{e}-02$ \\
26 & 319 & 0.11645 & $5.8674 \mathrm{e}-04$ & $1.4676 \mathrm{e}-02$ \\
27 & 343 & 0.88553 & $5.8674 \mathrm{e}-04$ & $1.4676 \mathrm{e}-02$ \\
28 & 240 & 0.11348 & $5.8674 \mathrm{e}-04$ & $1.4676 \mathrm{e}-02$ \\
29 & 420 & 0.13627 & $5.8674 \mathrm{e}-04$ & $1.4676 \mathrm{e}-02$ \\
30 & 187 & 0.63875 & $5.8674 \mathrm{e}-04$ & $1.4676 \mathrm{e}-02$ \\
31 & 184 & 0.43062 & $5.8674 \mathrm{e}-04$ & $1.4676 \mathrm{e}-02$ \\
32 & 487 & 0.65461 & $5.8674 \mathrm{e}-04$ & $1.4676 \mathrm{e}-02$ \\
33 & 142 & 0.87661 & $5.8674 \mathrm{e}-04$ & $1.4676 \mathrm{e}-02$ \\
34 & 463 & 0.73389 & $5.8674 \mathrm{e}-04$ & $1.4676 \mathrm{e}-02$ \\
35 & 307 & 0.60505 & $5.8674 \mathrm{e}-04$ & $1.4676 \mathrm{e}-02$ \\
36 & 476 & 0.64371 & $5.8674 \mathrm{e}-04$ & $1.4676 \mathrm{e}-02$ \\
37 & 353 & 0.78345 & $5.8674 \mathrm{e}-04$ & $1.4676 \mathrm{e}-02$ \\
38 & 30 & 0.67047 & $5.8674 \mathrm{e}-04$ & $1.4676 \mathrm{e}-02$ \\
39 & 267 & 0.15213 & $5.8674 \mathrm{e}-04$ & $1.4676 \mathrm{e}-02$ \\
40 & 20 & 0.96085 & $5.8674 \mathrm{e}-04$ & $1.4676 \mathrm{e}-02$ \\
\hline
\end{tabular}


TABLE $4 . n=1009$ and $\gamma_{j}=0.75^{j}$

\begin{tabular}{|r|r|l|l|c|}
\hline$d$ & $z_{d}$ & $\Delta_{d}$ & $e_{n, d}(\boldsymbol{z}, \boldsymbol{\Delta})$ & $E_{n, d}$ \\
\hline 1 & 1 & 0.50099 & $2.4777 \mathrm{e}-04$ & $1.1130 \mathrm{e}-02$ \\
2 & 282 & 0.49108 & $5.3445 \mathrm{e}-04$ & $1.6579 \mathrm{e}-02$ \\
3 & 374 & 0.34242 & $8.9897 \mathrm{e}-04$ & $2.0888 \mathrm{e}-02$ \\
4 & 153 & 0.77750 & $1.3495 \mathrm{e}-03$ & $2.4368 \mathrm{e}-02$ \\
5 & 135 & 0.60307 & $1.7838 \mathrm{e}-03$ & $2.7159 \mathrm{e}-02$ \\
6 & 195 & 0.31665 & $2.1858 \mathrm{e}-03$ & $2.9371 \mathrm{e}-02$ \\
7 & 209 & 0.84787 & $2.5536 \mathrm{e}-03$ & $3.1103 \mathrm{e}-02$ \\
8 & 232 & 0.75173 & $2.8540 \mathrm{e}-03$ & $3.2446 \mathrm{e}-02$ \\
9 & 349 & 0.64172 & $3.1148 \mathrm{e}-03$ & $3.3480 \mathrm{e}-02$ \\
10 & 386 & 0.55946 & $3.3418 \mathrm{e}-03$ & $3.4271 \mathrm{e}-02$ \\
11 & 99 & 0.25917 & $3.5119 \mathrm{e}-03$ & $3.4873 \mathrm{e}-02$ \\
12 & 219 & 0.56145 & $3.6422 \mathrm{e}-03$ & $3.5330 \mathrm{e}-02$ \\
13 & 170 & 0.94698 & $3.7449 \mathrm{e}-03$ & $3.5675 \mathrm{e}-02$ \\
14 & 41 & 0.96283 & $3.8262 \mathrm{e}-03$ & $3.5936 \mathrm{e}-02$ \\
15 & 343 & 0.12438 & $3.8913 \mathrm{e}-03$ & $3.6132 \mathrm{e}-02$ \\
16 & 319 & 0.83399 & $3.9394 \mathrm{e}-03$ & $3.6280 \mathrm{e}-02$ \\
17 & 439 & 0.93508 & $3.9774 \mathrm{e}-03$ & $3.6392 \mathrm{e}-02$ \\
18 & 359 & 0.07284 & $4.0061 \mathrm{e}-03$ & $3.6475 \mathrm{e}-02$ \\
19 & 247 & 0.44945 & $4.0282 \mathrm{e}-03$ & $3.6538 \mathrm{e}-02$ \\
20 & 412 & 0.14222 & $4.0446 \mathrm{e}-03$ & $3.6585 \mathrm{e}-02$ \\
21 & 240 & 0.18186 & $4.0573 \mathrm{e}-03$ & $3.6620 \mathrm{e}-02$ \\
22 & 256 & 0.83994 & $4.0666 \mathrm{e}-03$ & $3.6647 \mathrm{e}-02$ \\
23 & 276 & 0.03122 & $4.0740 \mathrm{e}-03$ & $3.6667 \mathrm{e}-02$ \\
24 & 442 & 0.60505 & $4.0797 \mathrm{e}-03$ & $3.6682 \mathrm{e}-02$ \\
25 & 380 & 0.11744 & $4.0840 \mathrm{e}-03$ & $3.6693 \mathrm{e}-02$ \\
26 & 53 & 0.92517 & $4.0872 \mathrm{e}-03$ & $3.6702 \mathrm{e}-02$ \\
27 & 216 & 0.09167 & $4.0896 \mathrm{e}-03$ & $3.6708 \mathrm{e}-02$ \\
28 & 69 & 0.78741 & $4.0914 \mathrm{e}-03$ & $3.6713 \mathrm{e}-02$ \\
29 & 467 & 0.20565 & $4.0928 \mathrm{e}-03$ & $3.6716 \mathrm{e}-02$ \\
30 & 228 & 0.73687 & $4.0938 \mathrm{e}-03$ & $3.6719 \mathrm{e}-02$ \\
31 & 420 & 0.02626 & $4.0946 \mathrm{e}-03$ & $3.6721 \mathrm{e}-02$ \\
32 & 103 & 0.84688 & $4.0952 \mathrm{e}-03$ & $3.6722 \mathrm{e}-02$ \\
33 & 449 & 0.53568 & $4.0956 \mathrm{e}-03$ & $3.6723 \mathrm{e}-02$ \\
34 & 278 & 0.06194 & $4.0960 \mathrm{e}-03$ & $3.6724 \mathrm{e}-02$ \\
35 & 361 & 0.24331 & $4.0962 \mathrm{e}-03$ & $3.6725 \mathrm{e}-02$ \\
36 & 420 & 0.75471 & $4.0964 \mathrm{e}-03$ & $3.6725 \mathrm{e}-02$ \\
37 & 59 & 0.92418 & $4.0966 \mathrm{e}-03$ & $3.6726 \mathrm{e}-02$ \\
38 & 103 & 0.07978 & $4.0967 \mathrm{e}-03$ & $3.6726 \mathrm{e}-02$ \\
39 & 449 & 0.90139 & $4.0968 \mathrm{e}-03$ & $3.6726 \mathrm{e}-02$ \\
40 & 59 & 0.92418 & $4.0968 \mathrm{e}-03$ & $3.6726 \mathrm{e}-02$ \\
\hline
\end{tabular}


TABLE 5. $n=1009$ and $\gamma_{j}=0.9^{j}$

\begin{tabular}{|r|r|l|l|c|}
\hline$d$ & $z_{d}$ & $\Delta_{d}$ & $e_{n, d}(\boldsymbol{z}, \boldsymbol{\Delta})$ & $E_{n, d}$ \\
\hline 1 & 1 & 0.71209 & $2.7142 \mathrm{e}-04$ & $1.2193 \mathrm{e}-02$ \\
2 & 282 & 0.02131 & $6.6925 \mathrm{e}-04$ & $1.9565 \mathrm{e}-02$ \\
3 & 374 & 0.29386 & $1.3280 \mathrm{e}-03$ & $2.6854 \mathrm{e}-02$ \\
4 & 153 & 0.31070 & $2.3878 \mathrm{e}-03$ & $3.4353 \mathrm{e}-02$ \\
5 & 135 & 0.03419 & $3.7750 \mathrm{e}-03$ & $4.2101 \mathrm{e}-02$ \\
6 & 228 & 0.42765 & $5.5115 \mathrm{e}-03$ & $5.0062 \mathrm{e}-02$ \\
7 & 474 & 0.86174 & $7.6802 \mathrm{e}-03$ & $5.8172 \mathrm{e}-02$ \\
8 & 484 & 0.95689 & $1.0076 \mathrm{e}-02$ & $6.6354 \mathrm{e}-02$ \\
9 & 345 & 0.64272 & $1.2730 \mathrm{e}-02$ & $7.4529 \mathrm{e}-02$ \\
10 & 450 & 0.26214 & $1.5662 \mathrm{e}-02$ & $8.2624 \mathrm{e}-02$ \\
11 & 181 & 0.10258 & $1.8711 \mathrm{e}-02$ & $9.0571 \mathrm{e}-02$ \\
12 & 468 & 0.16799 & $2.1847 \mathrm{e}-02$ & $9.8311 \mathrm{e}-02$ \\
13 & 53 & 0.91328 & $2.5108 \mathrm{e}-02$ & $1.0579 \mathrm{e}-01$ \\
14 & 86 & 0.78741 & $2.8271 \mathrm{e}-02$ & $1.1298 \mathrm{e}-01$ \\
15 & 247 & 0.50892 & $3.1342 \mathrm{e}-02$ & $1.1984 \mathrm{e}-01$ \\
16 & 295 & 0.38305 & $3.4296 \mathrm{e}-02$ & $1.2634 \mathrm{e}-01$ \\
17 & 58 & 0.99653 & $3.7238 \mathrm{e}-02$ & $1.3249 \mathrm{e}-01$ \\
18 & 32 & 0.45937 & $4.0014 \mathrm{e}-02$ & $1.3827 \mathrm{e}-01$ \\
19 & 99 & 0.00347 & $4.2744 \mathrm{e}-02$ & $1.4367 \mathrm{e}-01$ \\
20 & 330 & 0.78741 & $4.5262 \mathrm{e}-02$ & $1.4871 \mathrm{e}-01$ \\
21 & 324 & 0.51784 & $4.7765 \mathrm{e}-02$ & $1.5340 \mathrm{e}-01$ \\
22 & 20 & 0.63578 & $5.0139 \mathrm{e}-02$ & $1.5774 \mathrm{e}-01$ \\
23 & 236 & 0.22646 & $5.2310 \mathrm{e}-02$ & $1.6175 \mathrm{e}-01$ \\
24 & 420 & 0.68236 & $5.4341 \mathrm{e}-02$ & $1.6544 \mathrm{e}-01$ \\
25 & 380 & 0.84192 & $5.6247 \mathrm{e}-02$ & $1.6884 \mathrm{e}-01$ \\
26 & 492 & 0.94896 & $5.8020 \mathrm{e}-02$ & $1.7195 \mathrm{e}-01$ \\
27 & 223 & 0.53370 & $5.9667 \mathrm{e}-02$ & $1.7480 \mathrm{e}-01$ \\
28 & 272 & 0.48216 & $6.1185 \mathrm{e}-02$ & $1.7741 \mathrm{e}-01$ \\
29 & 438 & 0.67740 & $6.2582 \mathrm{e}-02$ & $1.7979 \mathrm{e}-01$ \\
30 & 157 & 0.91526 & $6.3863 \mathrm{e}-02$ & $1.8195 \mathrm{e}-01$ \\
31 & 145 & 0.54856 & $6.5043 \mathrm{e}-02$ & $1.8393 \mathrm{e}-01$ \\
32 & 355 & 0.28295 & $6.6100 \mathrm{e}-02$ & $1.8572 \mathrm{e}-01$ \\
33 & 496 & 0.24727 & $6.7058 \mathrm{e}-02$ & $1.8735 \mathrm{e}-01$ \\
34 & 383 & 0.27304 & $6.7960 \mathrm{e}-02$ & $1.8883 \mathrm{e}-01$ \\
35 & 132 & 0.36422 & $6.8774 \mathrm{e}-02$ & $1.9017 \mathrm{e}-01$ \\
36 & 197 & 0.61893 & $6.9520 \mathrm{e}-02$ & $1.9139 \mathrm{e}-01$ \\
37 & 309 & 0.16105 & $7.0197 \mathrm{e}-02$ & $1.9249 \mathrm{e}-01$ \\
38 & 257 & 0.58028 & $7.0808 \mathrm{e}-02$ & $1.9348 \mathrm{e}-01$ \\
39 & 231 & 0.04410 & $7.1366 \mathrm{e}-02$ & $1.9438 \mathrm{e}-01$ \\
40 & 125 & 0.01140 & $7.1877 \mathrm{e}-02$ & $1.9520 \mathrm{e}-01$ \\
\hline
\end{tabular}


TABLE 6. $n=2003$ and $\gamma_{j}=1 / j^{2}$

\begin{tabular}{|r|r|l|l|c|}
\hline$d$ & $z_{d}$ & $\Delta_{d}$ & $e_{n, d}(\boldsymbol{z}, \boldsymbol{\Delta})$ & $E_{n, d}$ \\
\hline 1 & 1 & 0.88442 & $1.4412 \mathrm{e}-04$ & $9.1219 \mathrm{e}-03$ \\
2 & 765 & 0.38168 & $2.3147 \mathrm{e}-04$ & $1.1016 \mathrm{e}-02$ \\
3 & 605 & 0.32277 & $2.9468 \mathrm{e}-04$ & $1.1893 \mathrm{e}-02$ \\
4 & 426 & 0.00674 & $3.4097 \mathrm{e}-04$ & $1.2396 \mathrm{e}-02$ \\
5 & 830 & 0.49451 & $3.7425 \mathrm{e}-04$ & $1.2721 \mathrm{e}-02$ \\
6 & 706 & 0.72067 & $4.0119 \mathrm{e}-04$ & $1.2948 \mathrm{e}-02$ \\
7 & 636 & 0.61882 & $4.2358 \mathrm{e}-04$ & $1.3115 \mathrm{e}-02$ \\
8 & 260 & 0.43660 & $4.4158 \mathrm{e}-04$ & $1.3244 \mathrm{e}-02$ \\
9 & 882 & 0.54044 & $4.5698 \mathrm{e}-04$ & $1.3346 \mathrm{e}-02$ \\
10 & 524 & 0.04768 & $4.6973 \mathrm{e}-04$ & $1.3428 \mathrm{e}-02$ \\
11 & 226 & 0.59386 & $4.8036 \mathrm{e}-04$ & $1.3497 \mathrm{e}-02$ \\
12 & 137 & 0.45956 & $4.8950 \mathrm{e}-04$ & $1.3554 \mathrm{e}-02$ \\
13 & 457 & 0.86246 & $4.9760 \mathrm{e}-04$ & $1.3603 \mathrm{e}-02$ \\
14 & 927 & 0.37419 & $5.0462 \mathrm{e}-04$ & $1.3646 \mathrm{e}-02$ \\
15 & 866 & 0.65776 & $5.1099 \mathrm{e}-04$ & $1.3683 \mathrm{e}-02$ \\
16 & 388 & 0.74114 & $5.1687 \mathrm{e}-04$ & $1.3715 \mathrm{e}-02$ \\
17 & 580 & 0.23640 & $5.2216 \mathrm{e}-04$ & $1.3744 \mathrm{e}-02$ \\
18 & 961 & 0.05467 & $5.2697 \mathrm{e}-04$ & $1.3770 \mathrm{e}-02$ \\
19 & 450 & 0.83899 & $5.3168 \mathrm{e}-04$ & $1.3793 \mathrm{e}-02$ \\
20 & 623 & 0.85397 & $5.3582 \mathrm{e}-04$ & $1.3813 \mathrm{e}-02$ \\
21 & 392 & 0.86445 & $5.3975 \mathrm{e}-04$ & $1.3832 \mathrm{e}-02$ \\
22 & 442 & 0.14703 & $5.4343 \mathrm{e}-04$ & $1.3849 \mathrm{e}-02$ \\
23 & 368 & 0.07314 & $5.4658 \mathrm{e}-04$ & $1.3865 \mathrm{e}-02$ \\
24 & 627 & 0.22641 & $5.4966 \mathrm{e}-04$ & $1.3880 \mathrm{e}-02$ \\
25 & 551 & 0.57688 & $5.5249 \mathrm{e}-04$ & $1.3893 \mathrm{e}-02$ \\
26 & 819 & 0.14653 & $5.5515 \mathrm{e}-04$ & $1.3905 \mathrm{e}-02$ \\
27 & 206 & 0.54943 & $5.5767 \mathrm{e}-04$ & $1.3917 \mathrm{e}-02$ \\
28 & 319 & 0.44508 & $5.6006 \mathrm{e}-04$ & $1.3927 \mathrm{e}-02$ \\
29 & 657 & 0.96430 & $5.6221 \mathrm{e}-04$ & $1.3937 \mathrm{e}-02$ \\
30 & 215 & 0.82252 & $5.6430 \mathrm{e}-04$ & $1.3947 \mathrm{e}-02$ \\
31 & 814 & 0.40664 & $5.6630 \mathrm{e}-04$ & $1.3955 \mathrm{e}-02$ \\
32 & 305 & 0.07614 & $5.6815 \mathrm{e}-04$ & $1.3963 \mathrm{e}-02$ \\
33 & 265 & 0.37868 & $5.6994 \mathrm{e}-04$ & $1.3971 \mathrm{e}-02$ \\
34 & 84 & 0.49101 & $5.7157 \mathrm{e}-04$ & $1.3978 \mathrm{e}-02$ \\
35 & 328 & 0.60934 & $5.7316 \mathrm{e}-04$ & $1.3985 \mathrm{e}-02$ \\
36 & 720 & 0.49451 & $5.7468 \mathrm{e}-04$ & $1.3992 \mathrm{e}-02$ \\
37 & 748 & 0.07564 & $5.7613 \mathrm{e}-04$ & $1.3998 \mathrm{e}-02$ \\
38 & 192 & 0.88193 & $5.7755 \mathrm{e}-04$ & $1.4003 \mathrm{e}-02$ \\
39 & 481 & 0.73365 & $5.7891 \mathrm{e}-04$ & $1.4009 \mathrm{e}-02$ \\
40 & 297 & 0.83749 & $5.8021 \mathrm{e}-04$ & $1.4014 \mathrm{e}-02$ \\
\hline
\end{tabular}


TABLE 7. $n=2003$ and $\gamma_{j}=0.5^{j}$

\begin{tabular}{|r|r|l|l|c|}
\hline$d$ & $z_{d}$ & $\Delta_{d}$ & $e_{n, d}(\boldsymbol{z}, \boldsymbol{\Delta})$ & $E_{n, d}$ \\
\hline 1 & 1 & 0.89141 & $1.0191 \mathrm{e}-04$ & $6.4501 \mathrm{e}-03$ \\
2 & 765 & 0.81653 & $1.7143 \mathrm{e}-04$ & $8.4305 \mathrm{e}-03$ \\
3 & 699 & 0.98278 & $2.2961 \mathrm{e}-04$ & $9.4160 \mathrm{e}-03$ \\
4 & 426 & 0.62232 & $2.6615 \mathrm{e}-04$ & $9.9136 \mathrm{e}-03$ \\
5 & 628 & 0.56890 & $2.8656 \mathrm{e}-04$ & $1.0164 \mathrm{e}-02$ \\
6 & 842 & 0.40664 & $2.9862 \mathrm{e}-04$ & $1.0290 \mathrm{e}-02$ \\
7 & 961 & 0.29581 & $3.0522 \mathrm{e}-04$ & $1.0353 \mathrm{e}-02$ \\
8 & 266 & 0.81553 & $3.0885 \mathrm{e}-04$ & $1.0384 \mathrm{e}-02$ \\
9 & 194 & 0.17898 & $3.1078 \mathrm{e}-04$ & $1.0400 \mathrm{e}-02$ \\
10 & 591 & 0.51648 & $3.1179 \mathrm{e}-04$ & $1.0408 \mathrm{e}-02$ \\
11 & 824 & 0.47654 & $3.1230 \mathrm{e}-04$ & $1.0412 \mathrm{e}-02$ \\
12 & 448 & 0.92836 & $3.1256 \mathrm{e}-04$ & $1.0414 \mathrm{e}-02$ \\
13 & 541 & 0.11807 & $3.1270 \mathrm{e}-04$ & $1.0415 \mathrm{e}-02$ \\
14 & 882 & 0.82601 & $3.1277 \mathrm{e}-04$ & $1.0416 \mathrm{e}-02$ \\
15 & 493 & 0.13704 & $3.1280 \mathrm{e}-04$ & $1.0416 \mathrm{e}-02$ \\
16 & 150 & 0.51498 & $3.1282 \mathrm{e}-04$ & $1.0416 \mathrm{e}-02$ \\
17 & 819 & 0.13205 & $3.1283 \mathrm{e}-04$ & $1.0416 \mathrm{e}-02$ \\
18 & 442 & 0.90190 & $3.1283 \mathrm{e}-04$ & $1.0416 \mathrm{e}-02$ \\
19 & 260 & 0.56390 & $3.1283 \mathrm{e}-04$ & $1.0416 \mathrm{e}-02$ \\
20 & 819 & 0.74513 & $3.1283 \mathrm{e}-04$ & $1.0416 \mathrm{e}-02$ \\
21 & 260 & 0.16051 & $3.1283 \mathrm{e}-04$ & $1.0416 \mathrm{e}-02$ \\
22 & 377 & 0.50200 & $3.1283 \mathrm{e}-04$ & $1.0416 \mathrm{e}-02$ \\
23 & 933 & 0.18797 & $3.1284 \mathrm{e}-04$ & $1.0416 \mathrm{e}-02$ \\
24 & 317 & 0.54793 & $3.1284 \mathrm{e}-04$ & $1.0416 \mathrm{e}-02$ \\
25 & 814 & 0.41513 & $3.1284 \mathrm{e}-04$ & $1.0416 \mathrm{e}-02$ \\
26 & 634 & 0.91338 & $3.1284 \mathrm{e}-04$ & $1.0416 \mathrm{e}-02$ \\
27 & 318 & 0.11608 & $3.1284 \mathrm{e}-04$ & $1.0416 \mathrm{e}-02$ \\
28 & 856 & 0.18847 & $3.1284 \mathrm{e}-04$ & $1.0416 \mathrm{e}-02$ \\
29 & 185 & 0.01173 & $3.1284 \mathrm{e}-04$ & $1.0416 \mathrm{e}-02$ \\
30 & 957 & 0.07014 & $3.1284 \mathrm{e}-04$ & $1.0416 \mathrm{e}-02$ \\
31 & 790 & 0.63929 & $3.1284 \mathrm{e}-04$ & $1.0416 \mathrm{e}-02$ \\
32 & 941 & 0.98677 & $3.1284 \mathrm{e}-04$ & $1.0416 \mathrm{e}-02$ \\
33 & 888 & 0.58088 & $3.1284 \mathrm{e}-04$ & $1.0416 \mathrm{e}-02$ \\
34 & 220 & 0.07564 & $3.1284 \mathrm{e}-04$ & $1.0416 \mathrm{e}-02$ \\
35 & 241 & 0.90339 & $3.1284 \mathrm{e}-04$ & $1.0416 \mathrm{e}-02$ \\
36 & 210 & 0.75462 & $3.1284 \mathrm{e}-04$ & $1.0416 \mathrm{e}-02$ \\
37 & 753 & 0.81103 & $3.1284 \mathrm{e}-04$ & $1.0416 \mathrm{e}-02$ \\
38 & 341 & 0.12257 & $3.1284 \mathrm{e}-04$ & $1.0416 \mathrm{e}-02$ \\
39 & 501 & 0.08762 & $3.1284 \mathrm{e}-04$ & $1.0416 \mathrm{e}-02$ \\
40 & 243 & 0.65477 & $3.1284 \mathrm{e}-04$ & $1.0416 \mathrm{e}-02$ \\
\hline
\end{tabular}


TABLE 8. $n=2003$ and $\gamma_{j}=0.75^{j}$

\begin{tabular}{|r|r|l|l|c|}
\hline$d$ & $z_{d}$ & $\Delta_{d}$ & $e_{n, d}(\boldsymbol{z}, \boldsymbol{\Delta})$ & $E_{n, d}$ \\
\hline 1 & 1 & 0.36021 & $1.2481 \mathrm{e}-04$ & $7.8998 \mathrm{e}-03$ \\
2 & 765 & 0.35821 & $2.7178 \mathrm{e}-04$ & $1.1767 \mathrm{e}-02$ \\
3 & 343 & 0.34723 & $4.8337 \mathrm{e}-04$ & $1.4825 \mathrm{e}-02$ \\
4 & 849 & 0.09860 & $7.1275 \mathrm{e}-04$ & $1.7295 \mathrm{e}-02$ \\
5 & 599 & 0.27134 & $9.9054 \mathrm{e}-04$ & $1.9276 \mathrm{e}-02$ \\
6 & 884 & 0.09561 & $1.2442 \mathrm{e}-03$ & $2.0846 \mathrm{e}-02$ \\
7 & 242 & 0.22841 & $1.4803 \mathrm{e}-03$ & $2.2075 \mathrm{e}-02$ \\
8 & 735 & 0.78507 & $1.6688 \mathrm{e}-03$ & $2.3029 \mathrm{e}-02$ \\
9 & 964 & 0.12956 & $1.8200 \mathrm{e}-03$ & $2.3763 \mathrm{e}-02$ \\
10 & 903 & 0.90639 & $1.9454 \mathrm{e}-03$ & $2.4324 \mathrm{e}-02$ \\
11 & 869 & 0.74513 & $2.0538 \mathrm{e}-03$ & $2.4751 \mathrm{e}-02$ \\
12 & 743 & 0.05667 & $2.1375 \mathrm{e}-03$ & $2.5075 \mathrm{e}-02$ \\
13 & 406 & 0.63929 & $2.2012 \mathrm{e}-03$ & $2.5321 \mathrm{e}-02$ \\
14 & 457 & 0.03120 & $2.2514 \mathrm{e}-03$ & $2.5506 \mathrm{e}-02$ \\
15 & 318 & 0.66026 & $2.2901 \mathrm{e}-03$ & $2.5645 \mathrm{e}-02$ \\
16 & 622 & 0.08163 & $2.3210 \mathrm{e}-03$ & $2.5750 \mathrm{e}-02$ \\
17 & 629 & 0.31727 & $2.3450 \mathrm{e}-03$ & $2.5829 \mathrm{e}-02$ \\
18 & 93 & 0.81902 & $2.3632 \mathrm{e}-03$ & $2.5888 \mathrm{e}-02$ \\
19 & 693 & 0.19046 & $2.3773 \mathrm{e}-03$ & $2.5933 \mathrm{e}-02$ \\
20 & 716 & 0.50150 & $2.3876 \mathrm{e}-03$ & $2.5966 \mathrm{e}-02$ \\
21 & 176 & 0.54194 & $2.3958 \mathrm{e}-03$ & $2.5991 \mathrm{e}-02$ \\
22 & 909 & 0.46855 & $2.4019 \mathrm{e}-03$ & $2.6010 \mathrm{e}-02$ \\
23 & 133 & 0.43460 & $2.4066 \mathrm{e}-03$ & $2.6024 \mathrm{e}-02$ \\
24 & 680 & 0.02272 & $2.4101 \mathrm{e}-03$ & $2.6035 \mathrm{e}-02$ \\
25 & 494 & 0.75312 & $2.4127 \mathrm{e}-03$ & $2.6043 \mathrm{e}-02$ \\
26 & 284 & 0.36071 & $2.4147 \mathrm{e}-03$ & $2.6049 \mathrm{e}-02$ \\
27 & 395 & 0.68173 & $2.4162 \mathrm{e}-03$ & $2.6053 \mathrm{e}-02$ \\
28 & 328 & 0.91288 & $2.4173 \mathrm{e}-03$ & $2.6057 \mathrm{e}-02$ \\
29 & 565 & 0.99875 & $2.4182 \mathrm{e}-03$ & $2.6059 \mathrm{e}-02$ \\
30 & 386 & 0.30879 & $2.4188 \mathrm{e}-03$ & $2.6061 \mathrm{e}-02$ \\
31 & 269 & 0.66775 & $2.4193 \mathrm{e}-03$ & $2.6063 \mathrm{e}-02$ \\
32 & 259 & 0.11707 & $2.4196 \mathrm{e}-03$ & $2.6064 \mathrm{e}-02$ \\
33 & 390 & 0.09611 & $2.4199 \mathrm{e}-03$ & $2.6064 \mathrm{e}-02$ \\
34 & 819 & 0.57389 & $2.4201 \mathrm{e}-03$ & $2.6065 \mathrm{e}-02$ \\
35 & 857 & 0.88492 & $2.4203 \mathrm{e}-03$ & $2.6065 \mathrm{e}-02$ \\
36 & 436 & 0.76261 & $2.4204 \mathrm{e}-03$ & $2.6066 \mathrm{e}-02$ \\
37 & 160 & 0.24938 & $2.4205 \mathrm{e}-03$ & $2.6066 \mathrm{e}-02$ \\
38 & 194 & 0.28233 & $2.4206 \mathrm{e}-03$ & $2.6066 \mathrm{e}-02$ \\
39 & 416 & 0.52247 & $2.4206 \mathrm{e}-03$ & $2.6066 \mathrm{e}-02$ \\
40 & 865 & 0.74713 & $2.4207 \mathrm{e}-03$ & $2.6067 \mathrm{e}-02$ \\
\hline
\end{tabular}


TABLE 9. $n=2003$ and $\gamma_{j}=0.9^{j}$

\begin{tabular}{|r|r|l|l|c|}
\hline$d$ & $z_{d}$ & $\Delta_{d}$ & $e_{n, d}(\boldsymbol{z}, \boldsymbol{\Delta})$ & $E_{n, d}$ \\
\hline 1 & 1 & 0.28882 & $1.3672 \mathrm{e}-04$ & $8.6538 \mathrm{e}-03$ \\
2 & 830 & 0.11058 & $3.4066 \mathrm{e}-04$ & $1.3887 \mathrm{e}-02$ \\
3 & 264 & 0.75562 & $7.1855 \mathrm{e}-04$ & $1.9060 \mathrm{e}-02$ \\
4 & 386 & 0.12007 & $1.2977 \mathrm{e}-03$ & $2.4382 \mathrm{e}-02$ \\
5 & 213 & 0.39066 & $2.1392 \mathrm{e}-03$ & $2.9881 \mathrm{e}-02$ \\
6 & 613 & 0.73764 & $3.2019 \mathrm{e}-03$ & $3.5532 \mathrm{e}-02$ \\
7 & 764 & 0.12356 & $4.5262 \mathrm{e}-03$ & $4.1287 \mathrm{e}-02$ \\
8 & 717 & 0.84798 & $6.0331 \mathrm{e}-03$ & $4.7094 \mathrm{e}-02$ \\
9 & 753 & 0.66775 & $7.7204 \mathrm{e}-03$ & $5.2897 \mathrm{e}-02$ \\
10 & 663 & 0.42062 & $9.5439 \mathrm{e}-03$ & $5.8643 \mathrm{e}-02$ \\
11 & 360 & 0.31628 & $1.1494 \mathrm{e}-02$ & $6.4283 \mathrm{e}-02$ \\
12 & 234 & 0.99176 & $1.3437 \mathrm{e}-02$ & $6.9776 \mathrm{e}-02$ \\
13 & 141 & 0.90090 & $1.5415 \mathrm{e}-02$ & $7.5086 \mathrm{e}-02$ \\
14 & 582 & 0.63230 & $1.7403 \mathrm{e}-02$ & $8.0186 \mathrm{e}-02$ \\
15 & 229 & 0.95681 & $1.9443 \mathrm{e}-02$ & $8.5053 \mathrm{e}-02$ \\
16 & 175 & 0.14503 & $2.1424 \mathrm{e}-02$ & $8.9672 \mathrm{e}-02$ \\
17 & 119 & 0.98577 & $2.3326 \mathrm{e}-02$ & $9.4034 \mathrm{e}-02$ \\
18 & 737 & 0.59985 & $2.5213 \mathrm{e}-02$ & $9.8134 \mathrm{e}-02$ \\
19 & 336 & 0.14953 & $2.7056 \mathrm{e}-02$ & $1.0197 \mathrm{e}-01$ \\
20 & 184 & 0.83749 & $2.8786 \mathrm{e}-02$ & $1.0555 \mathrm{e}-01$ \\
21 & 959 & 0.01423 & $3.0406 \mathrm{e}-02$ & $1.0887 \mathrm{e}-01$ \\
22 & 785 & 0.58987 & $3.1929 \mathrm{e}-02$ & $1.1195 \mathrm{e}-01$ \\
23 & 728 & 0.92586 & $3.3361 \mathrm{e}-02$ & $1.1480 \mathrm{e}-01$ \\
24 & 304 & 0.22591 & $3.4699 \mathrm{e}-02$ & $1.1742 \mathrm{e}-01$ \\
25 & 759 & 0.31677 & $3.5930 \mathrm{e}-02$ & $1.1983 \mathrm{e}-01$ \\
26 & 411 & 0.05367 & $3.7087 \mathrm{e}-02$ & $1.2204 \mathrm{e}-01$ \\
27 & 774 & 0.18747 & $3.8162 \mathrm{e}-02$ & $1.2407 \mathrm{e}-01$ \\
28 & 537 & 0.06715 & $3.9146 \mathrm{e}-02$ & $1.2592 \mathrm{e}-01$ \\
29 & 430 & 0.90939 & $4.0050 \mathrm{e}-02$ & $1.2760 \mathrm{e}-01$ \\
30 & 150 & 0.84948 & $4.0883 \mathrm{e}-02$ & $1.2914 \mathrm{e}-01$ \\
31 & 160 & 0.44758 & $4.1637 \mathrm{e}-02$ & $1.3054 \mathrm{e}-01$ \\
32 & 630 & 0.23789 & $4.2339 \mathrm{e}-02$ & $1.3182 \mathrm{e}-01$ \\
33 & 435 & 0.01023 & $4.2978 \mathrm{e}-02$ & $1.3297 \mathrm{e}-01$ \\
34 & 855 & 0.30979 & $4.3558 \mathrm{e}-02$ & $1.3402 \mathrm{e}-01$ \\
35 & 275 & 0.24938 & $4.4090 \mathrm{e}-02$ & $1.3498 \mathrm{e}-01$ \\
36 & 380 & 0.98078 & $4.4572 \mathrm{e}-02$ & $1.3584 \mathrm{e}-01$ \\
37 & 443 & 0.75911 & $4.5013 \mathrm{e}-02$ & $1.3662 \mathrm{e}-01$ \\
38 & 819 & 0.37319 & $4.5412 \mathrm{e}-02$ & $1.3733 \mathrm{e}-01$ \\
39 & 402 & 0.46355 & $4.5774 \mathrm{e}-02$ & $1.3796 \mathrm{e}-01$ \\
40 & 158 & 0.14054 & $4.6109 \mathrm{e}-02$ & $1.3854 \mathrm{e}-01$ \\
\hline
\end{tabular}


TABLE $10 . n=4001$ and $\gamma_{j}=1 / j^{2}$

\begin{tabular}{|r|r|l|l|c|}
\hline$d$ & $z_{d}$ & $\Delta_{d}$ & $e_{n, d}(\boldsymbol{z}, \boldsymbol{\Delta})$ & $E_{n, d}$ \\
\hline 1 & 1 & 0.34904 & $7.2123 \mathrm{e}-05$ & $6.4542 \mathrm{e}-03$ \\
2 & 1478 & 0.65946 & $1.1889 \mathrm{e}-04$ & $7.7941 \mathrm{e}-03$ \\
3 & 1180 & 0.97063 & $1.5244 \mathrm{e}-04$ & $8.4148 \mathrm{e}-03$ \\
4 & 1240 & 0.14609 & $1.7710 \mathrm{e}-04$ & $8.7705 \mathrm{e}-03$ \\
5 & 907 & 0.87966 & $1.9647 \mathrm{e}-04$ & $9.0005 \mathrm{e}-03$ \\
6 & 767 & 0.89365 & $2.1069 \mathrm{e}-04$ & $9.1611 \mathrm{e}-03$ \\
7 & 528 & 0.41427 & $2.2248 \mathrm{e}-04$ & $9.2797 \mathrm{e}-03$ \\
8 & 1563 & 0.73044 & $2.3276 \mathrm{e}-04$ & $9.3707 \mathrm{e}-03$ \\
9 & 1110 & 0.15434 & $2.4118 \mathrm{e}-04$ & $9.4428 \mathrm{e}-03$ \\
10 & 967 & 0.47976 & $2.4831 \mathrm{e}-04$ & $9.5012 \mathrm{e}-03$ \\
11 & 1399 & 0.55424 & $2.5461 \mathrm{e}-04$ & $9.5496 \mathrm{e}-03$ \\
12 & 557 & 0.47026 & $2.6045 \mathrm{e}-04$ & $9.5904 \mathrm{e}-03$ \\
13 & 936 & 0.50350 & $2.6541 \mathrm{e}-04$ & $9.6251 \mathrm{e}-03$ \\
14 & 325 & 0.21657 & $2.6985 \mathrm{e}-04$ & $9.6550 \mathrm{e}-03$ \\
15 & 1128 & 0.77993 & $2.7387 \mathrm{e}-04$ & $9.6811 \mathrm{e}-03$ \\
16 & 1430 & 0.31930 & $2.7728 \mathrm{e}-04$ & $9.7041 \mathrm{e}-03$ \\
17 & 1741 & 0.63522 & $2.8056 \mathrm{e}-04$ & $9.7245 \mathrm{e}-03$ \\
18 & 981 & 0.67896 & $2.8335 \mathrm{e}-04$ & $9.7426 \mathrm{e}-03$ \\
19 & 1592 & 0.01837 & $2.8594 \mathrm{e}-04$ & $9.7589 \mathrm{e}-03$ \\
20 & 576 & 0.14209 & $2.8830 \mathrm{e}-04$ & $9.7737 \mathrm{e}-03$ \\
21 & 1893 & 0.76768 & $2.9043 \mathrm{e}-04$ & $9.7870 \mathrm{e}-03$ \\
22 & 1786 & 0.51050 & $2.9251 \mathrm{e}-04$ & $9.7992 \mathrm{e}-03$ \\
23 & 1268 & 0.82542 & $2.9442 \mathrm{e}-04$ & $9.8103 \mathrm{e}-03$ \\
24 & 1213 & 0.45451 & $2.9626 \mathrm{e}-04$ & $9.8206 \mathrm{e}-03$ \\
25 & 1089 & 0.08960 & $2.9794 \mathrm{e}-04$ & $9.8300 \mathrm{e}-03$ \\
26 & 1793 & 0.92239 & $2.9955 \mathrm{e}-04$ & $9.8387 \mathrm{e}-03$ \\
27 & 881 & 0.73969 & $3.0104 \mathrm{e}-04$ & $9.8468 \mathrm{e}-03$ \\
28 & 1904 & 0.13284 & $3.0247 \mathrm{e}-04$ & $9.8543 \mathrm{e}-03$ \\
29 & 1721 & 0.92489 & $3.0383 \mathrm{e}-04$ & $9.8613 \mathrm{e}-03$ \\
30 & 1943 & 0.22282 & $3.0505 \mathrm{e}-04$ & $9.8679 \mathrm{e}-03$ \\
31 & 1766 & 0.28880 & $3.0623 \mathrm{e}-04$ & $9.8740 \mathrm{e}-03$ \\
32 & 1025 & 0.28280 & $3.0733 \mathrm{e}-04$ & $9.8798 \mathrm{e}-03$ \\
33 & 402 & 0.97688 & $3.0837 \mathrm{e}-04$ & $9.8852 \mathrm{e}-03$ \\
34 & 732 & 0.17183 & $3.0939 \mathrm{e}-04$ & $9.8903 \mathrm{e}-03$ \\
35 & 598 & 0.76018 & $3.1034 \mathrm{e}-04$ & $9.8952 \mathrm{e}-03$ \\
36 & 189 & 0.06236 & $3.1123 \mathrm{e}-04$ & $9.8997 \mathrm{e}-03$ \\
37 & 1775 & 0.46351 & $3.1211 \mathrm{e}-04$ & $9.9040 \mathrm{e}-03$ \\
38 & 1798 & 0.32579 & $3.1292 \mathrm{e}-04$ & $9.9081 \mathrm{e}-03$ \\
39 & 1729 & 0.18633 & $3.1370 \mathrm{e}-04$ & $9.9120 \mathrm{e}-03$ \\
40 & 1621 & 0.39878 & $3.1447 \mathrm{e}-04$ & $9.9157 \mathrm{e}-03$ \\
\hline
\end{tabular}


TABLE 11. $n=4001$ and $\gamma_{j}=0.5^{j}$

\begin{tabular}{|r|r|l|l|c|}
\hline$d$ & $z_{d}$ & $\Delta_{d}$ & $e_{n, d}(\boldsymbol{z}, \boldsymbol{\Delta})$ & $E_{n, d}$ \\
\hline 1 & 1 & 0.48625 & $5.0976 \mathrm{e}-05$ & $4.5638 \mathrm{e}-03$ \\
2 & 1478 & 0.31805 & $8.7832 \mathrm{e}-05$ & $5.9650 \mathrm{e}-03$ \\
3 & 823 & 0.46651 & $1.1778 \mathrm{e}-04$ & $6.6623 \mathrm{e}-03$ \\
4 & 1769 & 0.01487 & $1.3747 \mathrm{e}-04$ & $7.0143 \mathrm{e}-03$ \\
5 & 555 & 0.56723 & $1.4946 \mathrm{e}-04$ & $7.1916 \mathrm{e}-03$ \\
6 & 1737 & 0.68270 & $1.5663 \mathrm{e}-04$ & $7.2806 \mathrm{e}-03$ \\
7 & 981 & 0.73369 & $1.6048 \mathrm{e}-04$ & $7.3252 \mathrm{e}-03$ \\
8 & 719 & 0.98888 & $1.6255 \mathrm{e}-04$ & $7.3475 \mathrm{e}-03$ \\
9 & 1080 & 0.00987 & $1.6362 \mathrm{e}-04$ & $7.3587 \mathrm{e}-03$ \\
10 & 1128 & 0.72844 & $1.6415 \mathrm{e}-04$ & $7.3643 \mathrm{e}-03$ \\
11 & 639 & 0.44576 & $1.6443 \mathrm{e}-04$ & $7.3671 \mathrm{e}-03$ \\
12 & 775 & 0.92389 & $1.6457 \mathrm{e}-04$ & $7.3685 \mathrm{e}-03$ \\
13 & 1560 & 0.00637 & $1.6465 \mathrm{e}-04$ & $7.3692 \mathrm{e}-03$ \\
14 & 1237 & 0.45601 & $1.6469 \mathrm{e}-04$ & $7.3695 \mathrm{e}-03$ \\
15 & 910 & 0.63747 & $1.6470 \mathrm{e}-04$ & $7.3697 \mathrm{e}-03$ \\
16 & 1547 & 0.97738 & $1.6471 \mathrm{e}-04$ & $7.3698 \mathrm{e}-03$ \\
17 & 1563 & 0.95314 & $1.6472 \mathrm{e}-04$ & $7.3698 \mathrm{e}-03$ \\
18 & 1902 & 0.45476 & $1.6472 \mathrm{e}-04$ & $7.3698 \mathrm{e}-03$ \\
19 & 419 & 0.45201 & $1.6472 \mathrm{e}-04$ & $7.3698 \mathrm{e}-03$ \\
20 & 660 & 0.89215 & $1.6472 \mathrm{e}-04$ & $7.3699 \mathrm{e}-03$ \\
21 & 1391 & 0.01812 & $1.6472 \mathrm{e}-04$ & $7.3699 \mathrm{e}-03$ \\
22 & 597 & 0.82542 & $1.6472 \mathrm{e}-04$ & $7.3699 \mathrm{e}-03$ \\
23 & 1525 & 0.59123 & $1.6473 \mathrm{e}-04$ & $7.3699 \mathrm{e}-03$ \\
24 & 1548 & 0.28080 & $1.6473 \mathrm{e}-04$ & $7.3699 \mathrm{e}-03$ \\
25 & 1925 & 0.53349 & $1.6473 \mathrm{e}-04$ & $7.3699 \mathrm{e}-03$ \\
26 & 1607 & 0.23382 & $1.6473 \mathrm{e}-04$ & $7.3699 \mathrm{e}-03$ \\
27 & 1203 & 0.87641 & $1.6473 \mathrm{e}-04$ & $7.3699 \mathrm{e}-03$ \\
28 & 705 & 0.04211 & $1.6473 \mathrm{e}-04$ & $7.3699 \mathrm{e}-03$ \\
29 & 1464 & 0.10635 & $1.6473 \mathrm{e}-04$ & $7.3699 \mathrm{e}-03$ \\
30 & 679 & 0.19383 & $1.6473 \mathrm{e}-04$ & $7.3699 \mathrm{e}-03$ \\
31 & 473 & 0.39128 & $1.6473 \mathrm{e}-04$ & $7.3699 \mathrm{e}-03$ \\
32 & 1146 & 0.83792 & $1.6473 \mathrm{e}-04$ & $7.3699 \mathrm{e}-03$ \\
33 & 1698 & 0.92089 & $1.6473 \mathrm{e}-04$ & $7.3699 \mathrm{e}-03$ \\
34 & 1295 & 0.14759 & $1.6473 \mathrm{e}-04$ & $7.3699 \mathrm{e}-03$ \\
35 & 602 & 0.47276 & $1.6473 \mathrm{e}-04$ & $7.3699 \mathrm{e}-03$ \\
36 & 1124 & 0.98013 & $1.6473 \mathrm{e}-04$ & $7.3699 \mathrm{e}-03$ \\
37 & 442 & 0.04186 & $1.6473 \mathrm{e}-04$ & $7.3699 \mathrm{e}-03$ \\
38 & 62 & 0.18033 & $1.6473 \mathrm{e}-04$ & $7.3699 \mathrm{e}-03$ \\
39 & 1003 & 0.60047 & $1.6473 \mathrm{e}-04$ & $7.3699 \mathrm{e}-03$ \\
40 & 427 & 0.43177 & $1.6473 \mathrm{e}-04$ & $7.3699 \mathrm{e}-03$ \\
\hline
\end{tabular}


TABLE 12. $n=4001$ and $\gamma_{j}=0.75^{j}$

\begin{tabular}{|r|r|l|l|c|}
\hline$d$ & $z_{d}$ & $\Delta_{d}$ & $e_{n, d}(\boldsymbol{z}, \boldsymbol{\Delta})$ & $E_{n, d}$ \\
\hline 1 & 1 & 0.32079 & $6.2470 \mathrm{e}-05$ & $5.5895 \mathrm{e}-03$ \\
2 & 1654 & 0.99263 & $1.4037 \mathrm{e}-04$ & $8.3258 \mathrm{e}-03$ \\
3 & 902 & 0.13684 & $2.5013 \mathrm{e}-04$ & $1.0489 \mathrm{e}-02$ \\
4 & 1195 & 0.40327 & $3.8181 \mathrm{e}-04$ & $1.2237 \mathrm{e}-02$ \\
5 & 1832 & 0.92864 & $5.3071 \mathrm{e}-04$ & $1.3639 \mathrm{e}-02$ \\
6 & 1741 & 0.35004 & $6.7705 \mathrm{e}-04$ & $1.4749 \mathrm{e}-02$ \\
7 & 280 & 0.07236 & $8.0454 \mathrm{e}-04$ & $1.5619 \mathrm{e}-02$ \\
8 & 629 & 0.40802 & $9.2128 \mathrm{e}-04$ & $1.6294 \mathrm{e}-02$ \\
9 & 1246 & 0.17408 & $1.0230 \mathrm{e}-03$ & $1.6813 \mathrm{e}-02$ \\
10 & 497 & 0.16183 & $1.1048 \mathrm{e}-03$ & $1.7210 \mathrm{e}-02$ \\
11 & 590 & 0.31055 & $1.1699 \mathrm{e}-03$ & $1.7513 \mathrm{e}-02$ \\
12 & 1360 & 0.99863 & $1.2215 \mathrm{e}-03$ & $1.7742 \mathrm{e}-02$ \\
13 & 861 & 0.59423 & $1.2596 \mathrm{e}-03$ & $1.7915 \mathrm{e}-02$ \\
14 & 791 & 0.58473 & $1.2912 \mathrm{e}-03$ & $1.8046 \mathrm{e}-02$ \\
15 & 1937 & 0.12109 & $1.3153 \mathrm{e}-03$ & $1.8145 \mathrm{e}-02$ \\
16 & 764 & 0.82192 & $1.3340 \mathrm{e}-03$ & $1.8219 \mathrm{e}-02$ \\
17 & 119 & 0.87066 & $1.3480 \mathrm{e}-03$ & $1.8275 \mathrm{e}-02$ \\
18 & 663 & 0.63297 & $1.3587 \mathrm{e}-03$ & $1.8317 \mathrm{e}-02$ \\
19 & 1043 & 0.17758 & $1.3671 \mathrm{e}-03$ & $1.8349 \mathrm{e}-02$ \\
20 & 1767 & 0.90490 & $1.3733 \mathrm{e}-03$ & $1.8372 \mathrm{e}-02$ \\
21 & 1574 & 0.93789 & $1.3781 \mathrm{e}-03$ & $1.8390 \mathrm{e}-02$ \\
22 & 644 & 0.15434 & $1.3816 \mathrm{e}-03$ & $1.8403 \mathrm{e}-02$ \\
23 & 813 & 0.74144 & $1.3843 \mathrm{e}-03$ & $1.8413 \mathrm{e}-02$ \\
24 & 108 & 0.49800 & $1.3864 \mathrm{e}-03$ & $1.8421 \mathrm{e}-02$ \\
25 & 713 & 0.40777 & $1.3879 \mathrm{e}-03$ & $1.8427 \mathrm{e}-02$ \\
26 & 1464 & 0.58348 & $1.3891 \mathrm{e}-03$ & $1.8431 \mathrm{e}-02$ \\
27 & 531 & 0.29630 & $1.3899 \mathrm{e}-03$ & $1.8434 \mathrm{e}-02$ \\
28 & 1024 & 0.01137 & $1.3906 \mathrm{e}-03$ & $1.8436 \mathrm{e}-02$ \\
29 & 1448 & 0.60447 & $1.3911 \mathrm{e}-03$ & $1.8438 \mathrm{e}-02$ \\
30 & 352 & 0.32979 & $1.3915 \mathrm{e}-03$ & $1.8440 \mathrm{e}-02$ \\
31 & 220 & 0.17633 & $1.3918 \mathrm{e}-03$ & $1.8441 \mathrm{e}-02$ \\
32 & 1061 & 0.85791 & $1.3920 \mathrm{e}-03$ & $1.8441 \mathrm{e}-02$ \\
33 & 1163 & 0.84291 & $1.3922 \mathrm{e}-03$ & $1.8442 \mathrm{e}-02$ \\
34 & 1519 & 0.96513 & $1.3923 \mathrm{e}-03$ & $1.8442 \mathrm{e}-02$ \\
35 & 847 & 0.40552 & $1.3924 \mathrm{e}-03$ & $1.8443 \mathrm{e}-02$ \\
36 & 1952 & 0.38428 & $1.3925 \mathrm{e}-03$ & $1.8443 \mathrm{e}-02$ \\
37 & 1184 & 0.42702 & $1.3925 \mathrm{e}-03$ & $1.8443 \mathrm{e}-02$ \\
38 & 473 & 0.38703 & $1.3926 \mathrm{e}-03$ & $1.8443 \mathrm{e}-02$ \\
39 & 325 & 0.10860 & $1.3926 \mathrm{e}-03$ & $1.8443 \mathrm{e}-02$ \\
40 & 1668 & 0.30930 & $1.3926 \mathrm{e}-03$ & $1.8443 \mathrm{e}-02$ \\
\hline
\end{tabular}


TABLE 13. $n=4001$ and $\gamma_{j}=0.9^{j}$

\begin{tabular}{|r|r|l|l|c|}
\hline$d$ & $z_{d}$ & $\Delta_{d}$ & $e_{n, d}(\boldsymbol{z}, \boldsymbol{\Delta})$ & $E_{n, d}$ \\
\hline 1 & 1 & 0.72669 & $6.8440 \mathrm{e}-05$ & $6.1230 \mathrm{e}-03$ \\
2 & 1478 & 0.50375 & $1.7648 \mathrm{e}-04$ & $9.8254 \mathrm{e}-03$ \\
3 & 823 & 0.79068 & $3.7630 \mathrm{e}-04$ & $1.3486 \mathrm{e}-02$ \\
4 & 1769 & 0.61722 & $6.9564 \mathrm{e}-04$ & $1.7251 \mathrm{e}-02$ \\
5 & 555 & 0.52974 & $1.1830 \mathrm{e}-03$ & $2.1142 \mathrm{e}-02$ \\
6 & 527 & 0.72094 & $1.8229 \mathrm{e}-03$ & $2.5140 \mathrm{e}-02$ \\
7 & 901 & 0.91465 & $2.5524 \mathrm{e}-03$ & $2.9213 \mathrm{e}-02$ \\
8 & 1128 & 0.19133 & $3.4570 \mathrm{e}-03$ & $3.3322 \mathrm{e}-02$ \\
9 & 1065 & 0.96913 & $4.5148 \mathrm{e}-03$ & $3.7427 \mathrm{e}-02$ \\
10 & 1559 & 0.51575 & $5.6462 \mathrm{e}-03$ & $4.1493 \mathrm{e}-02$ \\
11 & 972 & 0.19208 & $6.8365 \mathrm{e}-03$ & $4.5483 \mathrm{e}-02$ \\
12 & 1444 & 0.75044 & $8.1036 \mathrm{e}-03$ & $4.9370 \mathrm{e}-02$ \\
13 & 366 & 0.97463 & $9.3827 \mathrm{e}-03$ & $5.3127 \mathrm{e}-02$ \\
14 & 109 & 0.61197 & $1.0681 \mathrm{e}-02$ & $5.6735 \mathrm{e}-02$ \\
15 & 1399 & 0.11260 & $1.1976 \mathrm{e}-02$ & $6.0179 \mathrm{e}-02$ \\
16 & 644 & 0.84341 & $1.3244 \mathrm{e}-02$ & $6.3448 \mathrm{e}-02$ \\
17 & 272 & 0.56348 & $1.4484 \mathrm{e}-02$ & $6.6534 \mathrm{e}-02$ \\
18 & 1422 & 0.49950 & $1.5674 \mathrm{e}-02$ & $6.9435 \mathrm{e}-02$ \\
19 & 955 & 0.89290 & $1.6816 \mathrm{e}-02$ & $7.2150 \mathrm{e}-02$ \\
20 & 608 & 0.45601 & $1.7900 \mathrm{e}-02$ & $7.4682 \mathrm{e}-02$ \\
21 & 1752 & 0.24356 & $1.8923 \mathrm{e}-02$ & $7.7034 \mathrm{e}-02$ \\
22 & 1170 & 0.51900 & $1.9906 \mathrm{e}-02$ & $7.9213 \mathrm{e}-02$ \\
23 & 140 & 0.34754 & $2.0829 \mathrm{e}-02$ & $8.1226 \mathrm{e}-02$ \\
24 & 384 & 0.52349 & $2.1684 \mathrm{e}-02$ & $8.3081 \mathrm{e}-02$ \\
25 & 1430 & 0.83117 & $2.2490 \mathrm{e}-02$ & $8.4786 \mathrm{e}-02$ \\
26 & 1391 & 0.67821 & $2.3244 \mathrm{e}-02$ & $8.6350 \mathrm{e}-02$ \\
27 & 335 & 0.95664 & $2.3941 \mathrm{e}-02$ & $8.7782 \mathrm{e}-02$ \\
28 & 1318 & 0.39778 & $2.4582 \mathrm{e}-02$ & $8.9091 \mathrm{e}-02$ \\
29 & 920 & 0.13184 & $2.5175 \mathrm{e}-02$ & $9.0286 \mathrm{e}-02$ \\
30 & 314 & 0.44576 & $2.5719 \mathrm{e}-02$ & $9.1374 \mathrm{e}-02$ \\
31 & 619 & 0.96338 & $2.6221 \mathrm{e}-02$ & $9.2366 \mathrm{e}-02$ \\
32 & 406 & 0.80042 & $2.6677 \mathrm{e}-02$ & $9.3267 \mathrm{e}-02$ \\
33 & 1547 & 0.99138 & $2.7097 \mathrm{e}-02$ & $9.4085 \mathrm{e}-02$ \\
34 & 295 & 0.81642 & $2.7476 \mathrm{e}-02$ & $9.4828 \mathrm{e}-02$ \\
35 & 1596 & 0.64246 & $2.7828 \mathrm{e}-02$ & $9.5501 \mathrm{e}-02$ \\
36 & 1160 & 0.28680 & $2.8151 \mathrm{e}-02$ & $9.6112 \mathrm{e}-02$ \\
37 & 881 & 0.03112 & $2.8445 \mathrm{e}-02$ & $9.6664 \mathrm{e}-02$ \\
38 & 1257 & 0.46826 & $2.8710 \mathrm{e}-02$ & $9.7164 \mathrm{e}-02$ \\
39 & 569 & 0.95889 & $2.8952 \mathrm{e}-02$ & $9.7616 \mathrm{e}-02$ \\
40 & 1627 & 0.72044 & $2.9169 \mathrm{e}-02$ & $9.8025 \mathrm{e}-02$ \\
\hline
\end{tabular}




\section{ACKNOWLEDGMENTS}

The support of the Australian Research Council is gratefully acknowledged, as are the valuable comments of H. Woźniakowski. Participants at the July $2000 \mathrm{Mt}$ Holyoke Summer Research Conference on Algorithms, Computational Complexity and Models of Computation, where a preliminary version of this paper was presented, encouraged the development of the fully discrete search over shifts in the version of the algorithm presented here.

\section{REFERENCES}

[1] Hickernell, F.J. (1998). A generalized discrepancy and quadrature error bound, Math. Comp., 67, 299-322. MR 98c:65032

[2] Hickernell, F. J. (1998). Lattice rules: How well do they measure up?, Random and QuasiRandom Point Sets (P. Hellekalek and G. Larcher, eds.), Lecture Notes in Statistics, vol. 138, Springer-Verlag, New York, 109-166. MR 2000b:65007

[3] Hickernell, F. J. and Woźniakowski, H. (2000). Integration and approximation in arbitrary dimensions, Adv. Comput. Math., 12, 25-58. MR 2001d:65007

[4] Novak, E. and Woźniakowski, H. (2001), Intractability results for integration and discrepancy, J. Complexity, 17, 388-441.

[5] Niederreiter, H. (1992). Random Number Generation and Quasi-Monte Carlo Methods, SIAM, Philadelphia. MR 93h:65008

[6] Sloan, I. H. and Joe, S. (1994). Lattice Methods for Multiple Integration, Clarendon Press, Oxford. MR 98a:65026

[7] Sloan, I. H. and Reztsov, A. V. (2002). Component-by-component construction of good lattice rules, Math. Comp., 17, 263-273.

[8] Sloan, I. H. and Woźniakowski, H. (1998). When are quasi-Monte Carlo algorithms efficient for high dimensional integrals?, J. Complexity, 14, 1-33. MR 99d:65184

[9] Sloan, I. H. and Woźniakowski, H. (2001). Tractability of multivariate integration for weighted Korobov classes, J. Complexity, 17, 697-721.

[10] Woźniakowski, H. (1999), Efficiency of quasi-Monte Carlo algorithms for high-dimensional integrals, Monte Carlo and Quasi-Monte Carlo Methods 1998 (H. Niederreiter and J. Spanier, eds.), Springer-Verlag, Berlin, 114-136.

School of Mathematics, University of New South Wales, Sydney, New South Wales 2052, Australia

E-mail address: sloan@maths.unsw.edu.au

Department of Mathematics, University of Waikato, Private Bag 3105, Hamilton, New ZeALAND

E-mail address: f.kuo@math.waikato.ac.nz

Department of Mathematics, University of Waikato, Private Bag 3105, Hamilton, New ZeALAND

E-mail address: stephenj@math.waikato.ac.nz 